\title{
A maximum stress at a distance criterion for the prediction of crack propagation in adhesively- bonded joints
}

\author{
Ph. Martiny ${ }^{a}$, F. Lani ${ }^{\text {b }}$, A.J. Kinloch ${ }^{c}$, T. Pardoen ${ }^{\text {b }}$ \\ ${ }^{a}$ Cenaero, Centre de Recherche en Aéronautique, \\ Rue des Frères Wright 29, B-6041 Gosselies, Belgium \\ ${ }^{b}$ Université catholique de Louvain, Institute of Mechanics, Materials and Civil Engineering, \\ Place Sainte Barbe 2, B-1348 Louvain-la-Neuve, Belgium \\ ${ }^{\mathrm{C} I m p e r i a l ~ C o l l e g e ~ L o n d o n, ~ M e c h a n i c a l ~ E n g i n e e r i n g ~ D e p a r t m e n t, ~}$ \\ Exhibition Road, London SW7 2AZ, UK
}

\begin{abstract}
The present work relates to the numerical prediction of the mode I failure of metal-to-metal adhesive joints under quasi-static, steady-state conditions by means of a criterion based on attaining a critical value of the maximum principal stress at a critical distance ahead of the crack tip. The model predicted very accurately the failure of three adhesives (i) over a wide range of the thickness of the adhesive layer from 0.1 to $1 \mathrm{~mm}$, and (ii) for two very different test geometries: namely the linear elastic fracture-mechanics tapered double-cantilever beam test and the elastic-plastic fracturemechanics wedge-peel test.
\end{abstract}

Keywords: adhesive joints; failure prediction; finite-element analysis; fracture mechanics; 


\section{Nomenclature}

\begin{tabular}{|c|c|}
\hline CZM & Cohesive zone model \\
\hline EPFM & Elastic-plastic fracture mechanics \\
\hline LEFM & Linear-elastic fracture-mechanics \\
\hline TDCB & Tapered double-cantilever beam \\
\hline$a$ & Crack length in the TDCB test \\
\hline$B$ & Width of the TDCB test specimens \\
\hline$E$ & Young modulus \\
\hline$E_{S}$ & Young modulus of the adherend material \\
\hline$G_{a}$ & Adhesive fracture energy \\
\hline$G_{a}^{r e f}$ & Reference adhesive fracture energy used to normalize the values $G_{a}$ \\
\hline$G_{a}^{S S Y}$ & Adhesive fracture energy under small-scale yielding conditions \\
\hline$h$ & Thickness of the adherends \\
\hline$h_{a d h}$ & Thickness of the adhesive layer \\
\hline$m$ & A parameter characterising the taper of the adherends in the TDCB test \\
\hline$n$ & Strain-hardening exponent in the power-hardening law \\
\hline$P$ & Load measured in the TDCB test \\
\hline$q$ & Hardening exponent in the Swift hardening law \\
\hline$r_{c}$ & Critical distance used in the failure criterion \\
\hline$r_{Y}$ & Height of the plastic zone above the crack plane \\
\hline$R_{1}$ & $\begin{array}{l}\text { Residual radius of curvature measured for the wedge-peel test for the arm to } \\
\text { which most of the adhesive remained attached after failure }\end{array}$ \\
\hline$R_{2}$ & $\begin{array}{l}\text { Residual radius of curvature measured for the wedge-peel test for the } \\
\text { arm closer to the crack plane }\end{array}$ \\
\hline$R_{a}$ & Average residual radius of curvature from the values $R_{1}$ and $R_{2}$ \\
\hline$\Gamma_{0}$ & Total energy dissipated in the cohesive zone in the CZM \\
\hline
\end{tabular}


Total energy dissipated in the adhesive layer

$\Gamma_{p} \quad$ Total plastic energy dissipated in the adhesive layer

$\Gamma_{p}^{A} \quad$ Total plastic energy dissipated in zone $\mathrm{A}$ in the adhesive layer

$\Gamma_{p}^{B} \quad$ Total plastic energy dissipated in zone B in the adhesive layer

$\Gamma_{p}^{C} \quad$ Total plastic energy dissipated in zone $C$ in the adhesive layer

Non-dimensional parameter in the Swift hardening-law

Initial yield stress in the Swift hardening-law

Poisson's ratio

$v_{s}$

Poisson's ratio of the adherend material

$\sigma_{0}$

Initial yield stress in the power-hardening law

$\sigma_{1}$

Maximum principal stress

$\sigma_{c}$

Critical maximum principal stress value used in the failure criterion

$\hat{\sigma}$

Peak stress used in the CZM 


\section{Introduction}

Adhesive bonding is a very widely employed method for joining materials, often referred to as the 'adherends'. Unlike other joining methods, such as riveting, welding or the use of mechanical fasteners, it offers quite a few major advantages [1] such (i) as the ability to join dissimilar materials and (ii) the fact that it gives a more uniform stress distribution across the joint, which in turn results in an increased service-life under cyclic-fatigue loading. However, adhesive bonding is still not widely used as the sole joining method in applications where the joint is a safety-critical feature of the structure. This can be explained by the lack of well-established numerical tools and design methodologies which result in high development costs.

Therefore, the present authors have been working on gaining a better understanding of the failure of adhesive joints and on developing reliable numerical models capable of predicting accurately this failure with a minimum number of characteristic, material parameters. To reduce the scope of the task, they have restricted themselves, as in the present paper, to metal-to-metal adhesive joints bonded using epoxy-based structural adhesives that fail under steady-state, mode I (or predominantly mode I) conditions and exhibit quasi-static crack growth. The model that the authors have used so far [2,3] has been derived from the work of Tvergaard and Hutchinson [4,5] and employs a cohesive zone model (CZM). The CZM represents the damage mechanisms responsible for fracture and has also been employed in modelling the failure of adhesive joints by, for example, Kafkalidis et al. [6] Yang et al. [7], Georgiou et al. [8], Ferracin [9], Pardoen at al. [10], Salomonsson and Andersson [11] and Cooper et al. [12]. In the model employed by Kafkalidis et al. [6], Yang et al. [7], Georgiou et al. [8] or Ferracin [9], the CZM represents the full thickness of the adhesive layer while, in the work of Salomonsson and Andersson [11], the polymer and the reinforcing particles are both represented by continuum finite elements that are all surrounded by interface elements, allowing for the development of micro-cracks. The approach followed by Pardoen at al. [10] and Martiny et al. [2,3] lies somewhere in between these two extremes. In their model, a single CZM is used which possesses zero height and has material parameters which define the shape and size of the CZM. The local fracture process is simulated by this cohesive zone and the local energy dissipation in the adhesive, ahead of the crack front, is accounted for by embedding the CZM between layers of elastic-plastic solid elements which represent the adhesive layer. A main feature of the model proposed by Martiny et al. [2,3] was that the values of these material parameters were held constant throughout the various modelling studies. The CZM was implemented in a twodimensional (2D) plane-strain, large-rotation, quasi-static, steady-state, finite-element formulation.

In Martiny et al. [2], the authors showed that the CZM was capable of predicting accurately the failure of adhesive joints consisting of aluminium-alloy adherends bonded together with an epoxybased adhesive, 'Bondmaster ESP 110', with an adhesive fracture energy, $G_{a}$, of the order of $1000 \mathrm{~J} / \mathrm{m}^{2}$. The values of the parameters that characterised the CZM of the adhesive were identified by using an elastic-plastic fracture-mechanics (EPFM) wedge-peel test configuration, coupled with an inverse-analysis method that was based on knowing the residual radii of curvature of the two adherend arms and the crack length. To validate the proposed numerical model it was used, together with the now-fixed CZM properties, to predict successfully the effect of various geometric features for other configurations of the elastic-plastic wedge-peel test, e.g. the effect of the thickness of the adhesive layer. The model was also successfully applied to fixed-arm peel tests, subjected to various peel angles. The numerical results from the proposed model were also post-processed to extract 
values of the adhesive fracture energy, $G_{a}$. For this particular adhesive, these values were found to be not significantly dependent upon the details of the peel test configuration, including the thickness, $h_{a d h}$, of the adhesive layer over a relatively very narrow range of 0.25 to $0.4 \mathrm{~mm}$. Also, the values of $G_{a}$ were in very good agreement with those measured using a linear-elastic fracturemechanics (LEFM) tapered double-cantilever beam (TDCB) test method. These observations were attributed to the fact that the main contribution to $G_{a}$ arose from the intrinsic work of fracture, $\Gamma_{0}$, i.e. the energy dissipated locally ahead of the crack tip by the damage mechanisms leading to fracture and as modelled by the CZM. As a corollary, it was recognised that the far-field plastic dissipation, $\Gamma_{p}$, occurring in the adhesive layer, but outside of the fracture process zone, was a second-order effect for the specific joints studied and loading configurations analysed.

In a following study, Martiny et al. [3], showed that the same model was capable of predicting accurately, with constant CZM parameters, the failure of adhesive joints made using a different epoxy-based adhesive, namely 'Dow Betamate 73455', with a far lower value of adhesive fracture energy in the range of 200-300 J/ $\mathrm{m}^{2}$ but now over a larger range of adhesive layer thicknesses, $h_{\text {adh }}$, from 0.1 to $1 \mathrm{~mm}$. These numerical modelling results revealed that the value of $G_{a}$ was a strong nonlinear function of the thickness of the adhesive layer. The other variables were shown to be of secondary importance in influencing the value of $G_{a}$, providing the adhesive did not contribute significantly to the bending stiffness of the joint. These results, which fully agreed with the experimental observations, were explained in detail by identifying and quantifying the different sources of energy dissipation in the adhesive layer contributing to the value of $G_{a}$. These sources were the locked-in elastic energy, crack tip plasticity, reverse plastic loading and plastic shear deformation at the adhesive/adherend interface, which when summed give the total energy dissipated, $\Gamma_{b}$, in the adhesive layer. Further, the magnitudes of these sources of energy dissipation were correlated to the degree of constraint at the crack tip, which was quantified by considering the opening angle of the cohesive zone at the crack tip.

Based on these results, the problem of predicting the behaviour of joints prepared using the relatively tough 'Bondmaster ESP 110' was revisited, in the context of the present study, but now when tested using the LEFM TDCB test method with a value of $h_{a d h}$ over the relatively wide range of 0.1 to $1 \mathrm{~mm}$. Unlike the results outlined above for the 'Dow Betamate 73455' adhesive (Martiny et al. [3]), modelling studies of the now more extensive experimental results were found to be to be unsuccessful, as shown and discussed in the Appendix. A major conclusion is that for the tougher 'ESP 110' adhesive the use of the CZM employing constant material parameters is only successful at predicting the variation of $G_{a}$ as a function of $h_{a d h}$ for a relatively narrow range of $h_{a d h}$ values. A tentative explanation for this limitation is that the damage mechanisms represented by the cohesive zone actually depend upon the value of $h_{a d h}$ via the level of constraint ahead of the crack tip and, more particularly, the stress triaxiality, as already reported in the case of metals (Siegmund and Brocks [13]; Pardoen at al. [14]. A possible solution would be to modify the cohesive zone parameter values as a function of the stress triaxiality, as recently suggested by Cooper et al. [12]. However, this requires performing multiple experiments to obtain the dependence of the cohesive zone parameters upon the stress state (Cooper et al. [12]) and can also an introduce intrinsic mesh dependency (e.g. Tvergaard and Hutchinson, [15]).

Therefore, in the present paper the authors have considered a somewhat different approach to the development of a suitable model for the failure of the adhesively-bonded joints and seek to use the 
attainment of a critical value of the (macroscopic) maximum principal stress at a critical distance ahead of the crack tip as the failure criterion. This failure criterion, proposed by Ritchie et al. [16] in the context of cleavage fracture in steel, has often since been used for very different applications and materials, see for example [16-20]. The key feature of this failure criterion is the introduction of a characteristic length which, in principle, is connected to the microstructure and damage phenomena. The advantage of this model is that it does not a priori partition the fracture energy between an 'intrinsic' energy dissipated in a cohesive zone and a ('less intrinsic') energy-term dissipated in the surrounding plastic zone(s). Indeed, such an arbitrary statement of the fracture process zones, their dimensions, and associated energies, can be difficult to define and justify in polymers.

The present paper is organised as follows. In Section 2, the different materials, tests and experimental results that are considered are briefly described. Section 3 gives more details about the model, its physical motivation, its implementation and the procedure that was followed to identify the different material parameters which are required in the maximum principal stress/critical distance model. In Section 4, the numerical predictions of the model obtained with these material parameters are critically compared to the experimental data. In Section 5, an overview is given, in a non-dimensional format, of the numerical predictions that can be obtained with such a model in terms of the adhesive fracture energy, $G_{a}$ as a function of the thickness, $h_{a d h}$ of the adhesive layer, as determined using LEFM TDCB tests. Finally, in Section 6, the main conclusions of the paper are given, and the generality and the limitations of the model are discussed.

\section{Materials and experimental procedures}

Three different epoxy-based structural adhesives, covering the range of $200-6500 \mathrm{~J} / \mathrm{m}^{2}$ in their values of adhesive fracture energy, $G_{a}$, have been studied. They are:

- Adhesive 'Betamate 73455', produced by Dow Automotive, which contains a large fraction of silica particles (Ferracin [9]) and exhibits values of $G_{a}$ of 200-300 J/m² for values of $h_{a d h}$ between 0.1 and $1 \mathrm{~mm}$;

- Adhesive 'ESP 110', produced by Bondmaster, which contains both aluminum flakes and rubber particles (da Silva and Adams [21]) and exhibits values of $G_{a}$ of $1000-1500 \mathrm{~J} / \mathrm{m}^{2}$ for values of $h_{\text {adh }}$ between 0.1 and $1 \mathrm{~mm}$;

- Adhesive 'Betamate 1493', produced by Dow Automotive, which contains a small volume fraction of small-size silica particles and as well as even smaller rubber particles (Ferracin [9]) and exhibits values of $G_{a}$ of $3500-6500 \mathrm{~J} / \mathrm{m}^{2}$ for values of $h_{a d h} 0.1$ and $1 \mathrm{~mm}$.

The elastic-plastic properties of each of these adhesives were determined in tension and compression using bulk specimens and their mode I fracture behaviour was studied employing joint specimens with different values of $h_{a d h}$, see Martiny et al. [2,3] for full details of the test procedures. Basically, two different test geometries and test methods were employed: the LEFM TDCB test (ISO Standard 25217:2009) as shown in Figure 1(a), and the EPFM wedge-peel test as shown in Figure $1(b)$. The fracture surfaces of the joint test specimens were also examined using a scanning electron microscope. 


\subsection{Adhesive 'Betamate 73455'}

Flat dumbbell-shaped specimens made of bulk adhesive 'Betamate $73455^{\prime}$ ' were tested in uniaxial tension at different strain-rates ranging from $2.5 \times 10^{-5}$ to $2.5 \times 10^{-2} \mathrm{~s}^{-1}$ (Ferracin [9]). Figure 2 shows the corresponding stress versus strain curves. It can be seen that the adhesive exhibits little ratedependence, little strain-hardening capacity and a relatively low fracture strain of below about $1 \%$.

The LEFM TDCB test specimens were prepared with different values of $h_{a d h}$ and with aluminiumalloy arms (grade 2014A) possessing a taper characterised by a value of $m$ equal to $2 \mathrm{~mm}^{-1}$ (ISO Standard 25217:2009). They were tested as described in Martiny et al. [3] using a cross-head speed of $0.1 \mathrm{~mm} / \mathrm{min}$. The value of $G_{a}$ was evaluated from the values of the crack length, $a$, and of the load, $P$, recorded over time during the tests, see Figure 1(a), via the 'corrected beam theory' (Blackman et al. [22]):

$$
G_{a}=\frac{4 P^{2}}{E_{S} B^{2}} m\left[1+0.43\left(\frac{3}{m a}\right)^{1 / 3}\right]
$$

where $B$ is the width of the specimen and $E_{S}$ is the modulus of the beam (or adherend) material. Some of the specimens failed with the crack propagating close to one of the adhesive/adherend interfaces whereas the others failed with the crack running close the centreline of the adhesive layer (Martiny et al. [2]). For the sake of consistency with the experimental results obtained on the two other adhesives, only the results pertaining to the crack propagating close the centreline of the adhesive layer were employed in the present study. The results showed no significant 'R-curve' behaviour and the $G_{a}$ values were independent of crack length values, $a$, between 120 and $210 \mathrm{~mm}$. The corresponding steady-state values of $G_{a}$ are given in Table 1 as a function of $h_{a d h}$. As in all the relevant tables, the standard deviations for the data are also given.

The EPFM wedge-peel test specimens were prepared with different thicknesses of the adhesive and with $0.78 \mathrm{~mm}$ thick mild-steel arms and were tested using a $1.8 \mathrm{~mm}$-thick wedge advancing at a speed of $10 \mathrm{~mm} / \mathrm{min}$ (Ferracin [9]). The residual radius of curvature of both arms, $R_{1}$ and $R_{2}$, were measured on each specimen after being removed from the testing machine. Most of the specimens failed with the crack propagating at a short distance from the centreline of the adhesive layer, so that both radii were in reasonably good agreement and hence could be readily combined together in a single, average value, $R_{a}$, according to:

$$
\frac{1}{R_{a}}=\left[\frac{1}{2}\left(\frac{1}{R_{1}^{n+1}}+\frac{1}{R_{2}^{n+1}}\right)\right]^{\frac{1}{n+1}}
$$

where $n$ is the strain-hardening exponent of the adherend material. The resulting radii values are given in Table 2 which shows that the thicker the adhesive layer, then the smaller the radius and, hence, the larger the value of $G_{a}$. Figure 3, obtained by Ferracin [9] using a scanning electron microscope, shows typical micrographs of the fracture surface of the wedge-peel test specimens. It can be seen from Figure $3(\mathrm{~b})$ that failure is accompanied by the cleavage of the silica particles which are of very different sizes (from $10 \mu \mathrm{m}$ to $200 \mu \mathrm{m}$ ) and which are separated by 20 to $200 \mu \mathrm{m}$, see Figure 3(a-b), depending on their size.

\subsection{Adhesive 'ESP 110'}

Round dumbbell-shaped specimens made of bulk adhesive 'ESP 110' were tested in uniaxial tension at different strain-rates ranging from $2 \times 10^{-5}$ to $2 \times 10^{-3} \mathrm{~s}^{-1}$ (Martiny et al. [2]). Figure 4 shows the 
corresponding stress versus strain curves. The adhesive exhibits little rate-dependence, some strainhardening capacity and a relatively small fracture strain of the order of $3 \%$. Compared to the stress versus strain curves shown in Figure 2, these curves reveal that the adhesive 'ESP 110' shows a similar modulus and a more ductile behaviour than the adhesive 'Betamate 73455', most likely due to the presence of the rubber particles.

LEFM TDCB test specimens were prepared with different values of $h_{\text {adh }}$ and with aluminium-alloy arms (grade 2014A) possessing a taper characterised by a value of $m$ equal to $2 \mathrm{~mm}^{-1}$, and were tested using a cross-head speed of $0.2 \mathrm{~mm} / \mathrm{min}$. The value of $G_{a}$ was evaluated as for the adhesive 'Betamate 73455'. All specimens failed with the crack propagating close to the centreline of the adhesive layer. The results showed no significant 'R-curve' behaviour and the values of $G_{a}$ were independent of crack length values, $a$, between 70 and $230 \mathrm{~mm}$. The corresponding steady-state values of $G_{a}$ are given in Table 3 which shows that the $G_{a}$ values for this adhesive are about five times larger than for the adhesive 'Betamate 73445 ', and vary from 1000 to $1500 \mathrm{~J} / \mathrm{m}^{2}$ as the value of $h_{a d h}$ is increased from 0.25 to $0.88 \mathrm{~mm}$. Figure 5 shows typical scanning electron micrographs of the adhesive 'ESP110'. Figure 5(a) is a polished section of the adhesive which clearly contains a significant amount of aluminium flakes, as characterised using energy-dispersive $\mathrm{X}$-ray analysis, which are about 30 to $100 \mu \mathrm{m}$ in size and which are separated by about 50 to $100 \mu \mathrm{m}$, see Figure $5(a)$. Figure $5(b)$ is the fracture surface of a TDCB test specimen and shows that failure is accompanied by debonding of the aluminium flakes.

EPFM wedge-peel test specimens were prepared with different values of $h_{\text {adh }}$ using the adhesive 'ESP 110' and with $1.00 \mathrm{~mm}$ and $1.45 \mathrm{~mm}$ thick aluminium-alloy arms (grade 5754-0) and were tested using a $1.5 \mathrm{~mm}$-thick wedge advancing at a speed of $7.5 \mathrm{~mm} / \mathrm{min}$ (Martiny et al. [2]). The residual radius of curvature of both arms was measured on each specimen after being removed from the testing machine. All specimens failed with the crack propagating close to the one of the adhesive/adherend interfaces so that the radius, $R_{1}$, measured on the arm to which most of the adhesive remained attached after failure was always several times larger than the radius, $R_{2}$, measured on the arm closer to the crack. As the latter radius showed less experimental scatter and gave a clearer indication of the energy expenditure in the adhesive, only the $R_{2}$ values were employed in the present study. These values are given in Table 4 which shows that (i) the thicker the adhesive layer for a given thickness of the arms, the smaller the radius and, hence, the larger the value of $G_{a}$ and (ii) the thicker the arms for a given adhesive layer thickness, then the larger the bending stiffness and, hence, the larger the radius.

\subsection{Adhesive 'Betamate 1493'}

Flat dumbbell-shaped specimens made of bulk adhesive 'Betamate 1493' were tested in uniaxial tension at strain-rates from $2.5 \times 10^{-5}$ to $2.5 \times 10^{-3} \mathrm{~s}^{-1}$ (Ferracin [9]) and cylindrical specimens made of bulk adhesive 'Betamate 1493' were tested in uniaxial compression at a strain-rate of about $10^{-3} \mathrm{~s}^{-1}$ (Georgiou [23]). Figure 6 shows the corresponding stress versus strain curves. It can be seen that, in tension, the adhesive exhibits rate-dependence, no hardening capacity and failure strains of the order of $5 \%$. On the other hand, in compression, the adhesive exhibits a relatively larger yield stress, a limited degree of strain-hardening and a far higher failure strain of the order of $30 \%$. By comparison with Figure 4, the stress versus strain curves in Figure 6 reveal that the adhesive 'Betamate 1493' possesses a lower modulus and exhibits a more ductile behaviour. 
LEFM TDCB test specimens were prepared with different values of $h_{\text {adh }}$ using the adhesive 'Betamate 1493', and with aluminium-alloy arms (grade 2014A) showing a taper characterised by a value of $m$ equal to $2 \mathrm{~mm}^{-1}$, and were tested using a cross-head speed of $0.1 \mathrm{~mm} / \mathrm{min}$ (Georgiou [23]). The value of the adhesive fracture energy was evaluated as for the adhesive 'Betamate 73455'. All the specimens failed with the crack propagating close to the centreline of the adhesive layer. The results showed no significant 'R-curve' behaviour and the $G_{a}$ values were independent of the crack length for values, $a$, between 90 and $180 \mathrm{~mm}$. The corresponding steady-state values of the values of $G_{a}$ are given in Table 5 which shows that the value of $G_{a}$ is approximately three to six times larger than that for the adhesive 'ESP 110 ' and increases from $3500 \mathrm{~J} / \mathrm{m}^{2}$ to $6500 \mathrm{~J} / \mathrm{m}^{2}$ as the value of $h_{\text {adh }}$ is increased from 0.25 to $0.6 \mathrm{~mm}$. These notably far higher values of $G_{a}$ are thought to originate from extensive plastic dissipation, via plastic void growth, in the epoxy polymer which may occur once cavitation of the rubber particles has taken place, especially in the case of the relatively ductile epoxy polymer used in the 'Betamate 1493' adhesive. Figure 7 shows typical scanning electron micrographs of the adhesive 'Betamate 1493'. Figure 7(a) is a polished section of the adhesive. The distribution of particles is more complicated to analyse compared to the adhesive 'ESP 110' in Figure 5(a), and no detailed quantification has been performed. Nevertheless, the presence of particles, probably silicates, with a large variation in sizes from several micrometres down to a few of hundred nanometres can be identified. The mean spacing is equal to a few micrometres. Figure $7(\mathrm{~b})$, which was obtained by Ferracin [9], shows typical scanning electron micrographs of the fracture surfaces of a TDCB test specimen. Crack propagation seems to be initiated by the micrometre-size particles leading to 'so-called' conical marks on the fracture surface.

EPFM wedge-peel test specimens prepared with different values of $h_{\text {adh }}$ using the adhesive 'Betamate 1493' and with $1.2 \mathrm{~mm}$-thick mild-steel arms (being the same grade as for the wedge-peel test specimens prepared with the adhesive 'Betamate 73455' in Section 2.1) were tested with a 1.8 $\mathrm{mm}$ thick wedge advancing at a speed of $10 \mathrm{~mm} / \mathrm{min}$ (Ferracin [9]). The residual radius of curvature of both arms, $R_{1}$ and $R_{2}$, were measured for each specimen after being removed from the testing machine. Most of the specimens reported here failed with the crack propagating at a short distance from the centreline of the adhesive layer so that both radii were reasonably close to each other in value, and hence were combined together to give a single, average value $R_{a}$ according to Equation (2). The resulting radii values are given in Table 6 which shows that the thicker the adhesive layer, the smaller the radius and, hence, the higher the adhesive fracture energy.

\section{The model}

\subsection{Fundamentals}

The present study focuses on conditions of predominantly mode I, quasi-static, steady-state crack propagation in the adhesive. The failure criterion that is proposed requires the maximum principal stress to reach a critical value, $\sigma_{c}$, at a fixed, critical distance, $r_{c}$, ahead of the crack tip: the values of $\sigma_{c}$ and $r_{c}$ being characteristic and constant for a given adhesive. This model belongs to the theory of critical distances, following the terminology widely used for many materials, for instance see Taylor [20] and the detailed review by Pardoen and Pineau [24] concerning the modelling of the cleavage fracture in steel. Of relevance to the present model is the previous work of Kinloch and Williams [18], Clarke and McGregor [19] and Crocombe et al. [25] who have reported the idea of using different 
stress (or strain) components or invariants, at a certain distance or averaged over a certain distance, to predict the onset of failure in either unprecracked or precracked joints.

To calculate efficiently, from the computational point-of-view, the stresses ahead of a crack propagating under steady-state conditions, a dedicated two-dimensional (2D) plane-strain, large deformation, quasi-static, steady-state finite element code was used. Plane-strain conditions were chosen due to the large width-to-thickness ratios exhibited by the specimens being studied. A formulation accounting for large deformation was necessary because of the large rotations that the peel arms of the wedge-peel test specimens could undergo. Inertia effects were neglected based on the low loading-rates being considered in the present study. Finally, a steady-state formulation was chosen, for the sake of computational efficiency, since it makes it possible to obtain the steady-state solution in a single calculation, whereas traditional transient schemes require propagating the crack over a sufficiently large number of time-steps, or equivalently, over a sufficiently large distance with respect to $h_{a d h}$ until steady-state conditions are attained

A more detailed description of this finite-element code may be found in Martiny et al. [2]. Essentially, as opposed to a classical, small-strain, transient finite element formulation, the large deformations are accounted for using a total Lagrangian formulation, based on the Green-Lagrange strain tensor and the second Piola-Kirchhoff stress tensor, and steady-state regime solutions are directly sought, following the finite-element methodology suggested by Dean and Hutchinson [26] and illustrated in Figure 9 , by imposing a uniform velocity field in the undeformed configuration $\left(X_{1}, X_{2}\right)$ equal to:

$$
\left\{\begin{array}{l}
V_{1}=-\dot{a} \\
V_{2}=0
\end{array}\right.
$$

where $\dot{a}$ is the constant crack velocity, which becomes, in the deformed configuration $\left(x_{1}, x_{2}\right)$ :

$$
\left\{\begin{array}{l}
v_{1}=\frac{\partial x_{1}}{\partial X_{i}} V_{i}=-\dot{a}\left(1+\frac{\partial u_{1}}{\partial X_{1}}\right) \\
v_{2}=\frac{\partial x_{2}}{\partial X_{i}} V_{i}=-\dot{a} \frac{\partial u_{2}}{\partial X_{1}}
\end{array}\right.
$$

Thus, in Figure 8, material is flowing through the mesh, from the right-hand side to the left-hand side, with the streamlines being at a tangent to the element edges aligned with the direction indicated by the arrows. The displacement of the nodes of the mesh remains the unknown factor in the finite-element problem but their values directly drive the velocity field via Equation (4). Contrary to more traditional transient formulations, the equations of plasticity are not integrated over time but over space, along the streamlines from far upstream where the material is undeformed and where the plastic strains are zero, down to the location of interest. This integration is readily performed in the undeformed configuration in which, see Figure $8(b)$, the streamlines are lines of constant $X_{2}$. In the present study, for the sake of simplicity, a modified Newton-Raphson scheme, which relies on the simple elastic-tangent operator, is used to solve the equations of equilibrium instead of the exact tangent operator, which would be far too complex to formulate.

\subsection{The wedge-peel test}

The EPFM wedge-peel test was modelled as described previously by Martiny et al. [3]. Referring to Figure 9, the position of the crack was imposed, as an approximation, to be along the centreline of the specimens to simulate the tests conducted on the adhesives 'Betamate 73455' and 'Betamate $1493^{\prime}$ in which the crack was experimentally observed to be propagating close to the centreline of 
the specimens. To simulate the tests conducted on the adhesive 'ESP 110', in which the crack was running close to one of the adhesive/adherend interfaces, the crack position was located at a distance of $25 \mu \mathrm{m}$ (i.e. $5-10 \%$ of the adhesive layer thickness) from the adhesive/adherend interface. (It is noteworthy that Kawashita et al. [27] measured a residual adhesive thickness of $65 \mu \mathrm{m}$ on similar specimens tested in the fixed-arm peel test.) To improve the predictive capabilities of the present model, it should ideally be further developed with the capability of predicting the locus of failure. An obvious approach would be to simulate different loci of failure and retain, among all of these options, the one that is the most energy-favourable. However, determining the failure path which requires the least energy for propagation is not straightforward. For example, as the crack moves away from the centreline of the specimens towards one of the adhesive/adherend interfaces, the energy expended in the adhesive above and below the crack plane varies as a function of both the amount of material available and the constraint imposed by the arms. Further, in the case of the EPFM wedge-peel test, the energy expended in bending in the adherend arms will then decrease in one arm whilst increasing in the other arm. Therefore, the crack path for which the total energy expended is minimum will be different for different joint configurations and geometric details, and thus is beyond the scope of the present paper.

Both the adhesive located on each side of the crack and the arms were meshed with second-order quadrilateral continuum elements which were assigned an elastic-plastic behaviour with isotropic hardening following the von Mises theory of plasticity. The nodes facing each other on both sides of the crack plane were rigidly tied together ahead of the crack tip by imposing the following multipoint constraints:

$$
\left\{\begin{array}{l}
u_{1}^{J}-u_{1}^{I}=0 \\
u_{2}^{J}-u_{2}^{I}=0
\end{array}\right.
$$

The presence of the wedge was taken into account by imposing the following multi-point constraint:

$$
u_{2}^{B}-u_{2}^{A}=D_{w}
$$

to the nodes A and B taken at a distance $a$ past the crack tip and, as an approximation, at the midthickness of the arms to avoid local plastic deformation in the adhesive. The length of the computational domain ahead of the crack tip, $l_{u}$, was chosen to be sufficiently large for the rightended section to be reasonably stress-free. The length of the domain past the wedge, $l_{d, t o t}$, was chosen to be sufficiently large to have a significant portion of the length, $l_{d, e x t}$, showing a uniform curvature and, hence, being relevant to the steady-state conditions being modelled. The crack length, $a$, was modified iteratively until the condition for continuing crack propagation was met, that is, until the maximum principal stress at a distance $r_{c}$ ahead of the crack tip was equal to $\sigma_{c}$, this stress being evaluated by spatial interpolation from the closest integration points of the continuum elements to the crack plane.

When imposing the position of the crack to lie along the centreline of the specimen, the problem becomes symmetric and the size of the problem can be reduced by modelling only the upper symmetrical half of the specimen. In that case, the node-tightening condition given in Equation (5) and the multi-point constraint in Equation (6), accounting for the presence of the wedge, become the simple displacement boundary conditions given in Equations (7) and (8) below: 


$$
\begin{aligned}
& u_{2}^{J}=0 \\
& u_{2}^{B}=\frac{1}{2} D_{w}
\end{aligned}
$$

Once found, the solution to the finite-element problem can be post-processed to calculate the residual radius of curvature of the arms and the value of the adhesive fracture energy. The residual radius of curvature was obtained by least-square fitting a circle, over the portion of $l_{d, \text { ext }}$ where steady-state conditions prevailed, through the positions $\left(x_{1}, x_{2}\right)$, in the deformed configuration, of the nodes located on the free surfaces of the arms. The term $G_{a}$ was numerically evaluated as the total external work per unit area of crack advance, $G_{t}$, minus the total energy expended in the substrates per unit area of crack advance, $G_{S}$ :

$$
G_{a}=G_{t}-G_{s}
$$

The total external work per unit area of crack advance was obtained by multiplying the nodal forces imposed by the wedge by the local material velocity divided by the crack velocity $\dot{a}$ :

$$
G_{t}=F_{i}^{A} \frac{v_{i}^{A}}{\dot{a}}+F_{i}^{B} \frac{v_{i}^{B}}{\dot{a}}
$$

which, making use of Equation (4) and realising that the multi-point constraint in Equation (6) only introduces vertical forces, becomes:

$$
G_{t}=-\left(\left.F_{2}^{A} \frac{\partial u_{2}}{\partial X_{1}}\right|_{A}+\left.F_{2}^{B} \frac{\partial u_{2}}{\partial X_{1}}\right|_{B}\right)
$$

Whereas the total energy expended in the substrates per unit area of crack advance, $G_{S}$, was obtained by integrating the material derivative of the strain energy density divided by the crack velocity $\dot{a}$, over the full thickness of both substrates (denoted by ' $s$ ' in the formulae below), from the right boundary (located at $X_{1}=X_{1}^{u}$ ) which is stress-free down to the location where $X_{1}=$ $X_{1}^{d}$ located anywhere in the portion of $l_{d, e x t}$ where steady-state conditions prevail:

$$
\begin{aligned}
G_{S} & =\frac{1}{\dot{a}} \int_{X_{1}^{d}}^{X_{1}^{u}} \int_{S} \frac{D W}{D t} d X_{2} d X_{1} \\
& =\frac{1}{\dot{a}} \int_{X_{1}^{d}}^{X_{1}^{u}} \int_{S} S_{i j}\left(\frac{\partial E_{i j}}{\partial t}+V_{k} \frac{\partial E_{i j}}{\partial X_{k}}\right) d X_{2} d X_{1}
\end{aligned}
$$

which, making use of the condition for a steady-state regime $(\partial / \partial t=0)$ as well as of Equation (3), becomes:

$$
G_{S}=-\int_{X_{1}^{d}}^{X_{1}^{u}} \int_{S} S_{i j} \frac{\partial E_{i j}}{\partial X_{1}} d X_{2} d X_{1}
$$

The accuracy of the numerical predictions was verified, for every configuration that was simulated in the present study, by conducting rigorous convergence studies. More particularly, the lengths $l_{u}$ and $l_{d, t o t}$ were successively made longer and the element sizes made smaller until the variations observed in the predicted values of the crack length, the residual radius of curvature and the adhesive fracture energy became negligible. These studies showed that the element size at the crack tip generally needed to be equal to $1 / 40 r_{c}$ or, depending on the thickness of the adhesive layer, equal to $1 / 40-1 / 800 h_{a d h}$ to obtain accurate results. The element sizes were progressively increased as one moved away from the crack tip. Using the aforementioned values, the meshes 
contained 75,000 to 300,000 nodes. This required total computational times ranging between five minutes and twenty minutes on eight processors. The values of the number of nodes and corresponding computational times discussed above should be understood as being the minimum needed to obtain accurate answers, and hence optimal values. Simulations were systematically run, as part of convergence studies, with finer meshes containing up to 3,000,000 nodes, which required up to 24 hours of computational time on eight processors. However, the results from such lengthy computational, and costly, runs did not show any significant changes in the values calculated. Thus, these studies verify the numerical accuracy of the results reported in the present paper.

\subsection{The TDCB test}

Steady-state conditions do not rigorously apply to the LEFM TDCB test specimens, since the thickness of the arms at right angles to the crack tip is constantly changing as the crack propagates. Nevertheless, the stress-state around the crack tip remains reasonably the same as the crack propagates, since the measured value of $G_{a}$ is essentially independent of the crack length, see Section 2. Therefore, the TDCB test can be represented using an equivalent steady-state model of the wedge-peel test, as explained by Martiny et al. [3]. Essentially, the TDCB test specimens were modelled in the present study with equivalent wedge-peel test specimens in which (i) the crack length was imposed to be equal to $200 \mathrm{~mm}$ (a value chosen arbitrarily in the range of crack lengths where reasonably constant $G_{a}$ values were measured in the TDCB test), (ii) the wedge thickness was iteratively modified until the resulting magnitude of the loading was such that the failure criterion was satisfied, (iii) the arm thickness, $h$, was iteratively modified until Equation (1) was satisfied. The details of the model and the post-processing operations are thus essentially the same as described in Section 3.2 for the EPFM wedge-peel test specimens, with the exception that for all the TDCB tests the crack location was along the centreline of the specimens.

\subsection{Identification of the material parameters of the model}

The different material properties that the model requires are: (i) the bulk elastic-plastic properties of the adherends (i.e. the Young's modulus, $E_{s}$, the Poisson ratio, $v_{S}$, and the strain-hardening law), the bulk elastic-plastic properties of the adhesive (i.e. the Young's modulus, $E$, the Poisson's ratio, $v$, and the strain-hardening law) and the failure criterion for the adhesive (i.e. the values of the maximum principal stress, $\sigma_{c}$, and the critical distance, $r_{c}$ ).

The aluminium-alloy grade (2014A) that was used to prepare the LEFM TDCB test specimens for the three adhesives was modelled as purely elastic, since no sign of plastic deformation was observed in the actual tests, i.e. the adherends all returned to their initial shape after unloading. The values of the Young's modulus and Poisson's ratio for the aluminium-alloy adherends, taken from Hadavinia et al. [28], are summarised in Table 7. The bulk elastic-plastic properties of all the other materials are also summarised in Table 7, and were directly measured as described above with the exception of the Poisson's ratios of the adhesives which were estimated from the literature. Martiny et al. [3] fitted the tensile stress versus strain curve of the mild-steel that was used to prepare the wedge-peel test specimens, as used for the adhesives 'Betamate 73455' and 'Betamate 1493', with the following equation:

$$
\sigma= \begin{cases}E \varepsilon & , \quad \varepsilon<\frac{\sigma_{0}}{E} \\ \sigma_{0}\left(\frac{E \varepsilon}{\sigma_{0}}\right)^{n}, & \varepsilon \geq \frac{\sigma_{0}}{E}\end{cases}
$$


which includes a power-law type of hardening. Martiny et al. [2] fitted the tensile stress versus strain curves of the aluminium-alloy grade (5754-0) that was used to prepare the EPFM wedge-peel test specimens for the adhesive 'ESP 110' with the following equation:

$$
\sigma= \begin{cases}E \varepsilon & , \quad \varepsilon<\frac{\kappa}{E} \\ \kappa\left[1+\eta\left(\varepsilon-\frac{\sigma}{E}\right)\right]^{q} & , \quad \varepsilon \geq \frac{\kappa}{E}\end{cases}
$$

which includes the so-called Swift hardening law.

The tensile stress versus strain curves of the adhesive 'Betamate 73455', in Figure 2, and of the adhesive 'ESP 110', in Figure 4, were also all fitted with Equation (15). The tensile stress versus strain curves of the adhesive 'Betamate 1493' given in Figure 6 did not cover the full range of strains that was expected to be encountered in the simulations of the corresponding adhesive joints, owing to the relatively high toughness values exhibited by this adhesive. Therefore, the compression stress versus strain curve was fitted instead, also using Equation (15). The resulting fit was then scaleddown, before being used in the modelling studies, to mirror the tensile curves at small strains and also account for the lower yield stress exhibited in tension by the material. As may be seen from Figures 2, 4 and 6, the results from these modelling studies represent a good fit to the experimental results. Finally, it should be noted that the value of $\sigma_{0}$ given from Equation (15) should be viewed in a similar manner as any other coefficient of the flow-law: its value was determined so as to give the best fit from a purely mathematical point of view, i.e. using a least-square regression analysis.

The material properties, $r_{c}$ and $\sigma_{c}$, which constitute the failure criterion were identified for each adhesive by an inverse analysis: their values were chosen so as to get the best possible agreement between the numerical predictions and the experimental data. Essentially, the EPFM wedge-peel and LEFM TDCB test configurations that were tested experimentally were modelled, as described above, to reproduce the experimental measurements that had been obtained. In the wedge-peel test simulations, the value of $a$ was chosen firstly so as to reproduce the minimum residual radius of curvature that had been measured and secondly so as to reproduce the maximum residual radius of curvature that had been measured, as the values of $h_{\text {adh }}$ or $h$ were changed. Similarly, in the TDCB test simulations, the load, or more precisely the wedge thickness in the equivalent wedge-peel test specimen, was chosen firstly to reproduce the minimum $G_{a}$ that had been measured and secondly to reproduce the maximum $G_{a}$ that had been measured, as the value of $h_{\text {adh }}$ was changed. All the corresponding possible values of the maximum principal stress as a function as the distance ahead of the crack tip were then plotted and the points $\left(r_{c}, \sigma_{c}\right)$ were searched for those that would be located between all the pairs of minimum and maximum curves pertaining to the different experimental, i.e. wedge-peel and TDCB, configurations tested. Figure 10 gives a simplified illustration of this procedure for the particular case of the adhesive 'ESP 110' in the sense that only two pairs of minimum and maximum curves are plotted: one for the TDCB test configuration corresponding to $h_{a d h}=0.32 \mathrm{~mm}$ and one for the wedge-peel test configuration corresponding to $h_{a d h}=0.25 \mathrm{~mm}$ and $h=1 \mathrm{~mm}$. The values of $r_{c}$ and $\sigma_{c}$ obtained from this procedure are given in Table 8 , together with their range of uncertainty. For the adhesive 'ESP 110', there was a finite region of the $\left(r_{c}, \sigma_{c}\right)$ space that was located between all the available pairs of minimum and maximum curves and the dimensions of this region determine the uncertainty on the values of $r_{c}$ and $\sigma_{c}$. For the two other adhesives, i.e. 'Betamate 73455 ' and 'Betamate 1493', it was not possible to find a finite region of the $\left(r_{c}, \sigma_{c}\right)$ space located between all the available pairs of minimum and maximum curves. 
However, different disconnected regions of the $\left(r_{c}, \sigma_{c}\right)$ space that were located between most of the available pairs of minimum and maximum curves could be readily found, with the exception of a few pairs that were different for each of these disconnected regions. The dimensions and the distance between these regions determine the uncertainty on the values of $r_{c}$ and $\sigma_{c}$ reported in Table 8. Finally, it was found that imposing the maximum principal stress to be equal to $\sigma_{c}$ at a critical distance ahead of the crack tip resulted in the observation that the value of the maximum principal local stress, $\sigma_{1}$, acting over this distance was such that always $\sigma_{c} \geq \sigma_{1}$.

\subsection{Physical interpretation of the parameters of the failure criterion}

Table 8 reports, along with the typical values of $r_{c}$, the sizes of the second-phase particles of silica or aluminium, and the typical spacing, that were identified from the micrographs shown previously. The values of $r_{c}$ are in good agreement with both the range of sizes of the inorganic particles present in the adhesive and with the range of particle spacings that were measured. From a conceptual point of view, this observation is in agreement with two possible physical interpretations of the model. The first interpretation is that for failure to occur, the (macroscopic) maximum principal stress needs to reach the critical value for void nucleation and/or micro-cracking to occur over a distance of at least equivalent to the size of the smallest particle. Indeed, this argument recognises the fact there are always particles positioned along the crack front, and which are relatively close to the crack tip, which may debond or fracture, and so assist in advancing the crack until the next potential damage site is reached. The second interpretation, which has been suggested in connection with the ductile fracture of metals, e.g. Xia and Shih [29] and Pardoen et al. [30,31], is that the length $r_{c}$ should reflect the particle spacing. This interpretation would also appear to be a reasonable agreement with the values shown in Table 8. However, the model used here is very simple, and, considering all the other complexities entering the real problem, e.g. finite-strain effects, local instabilities, local-softening effects, distribution effects, the presence of more than one length scale, an inhomogeneous stressstate in the matrix due to the presence of the particles, etc., the quantitative agreement between the identified and observed length-scales is very satisfactory. Thus, the proposed physical interpretations suggested above do indeed appear to reflect the major dominant physical mechanisms.

Considering the values of the critical maximum principal stress, $\sigma_{c}$, reported in Table 8 , then they represent an average stress that must be attained over a sufficiently large distance to allow the local stress on a particle to reach the true fracture stress of the particle or of the interface. Damage nucleation will proceed by the fracture or debonding of the particle giving rise to a rounded, or flat, initial void or micro-crack. Table 8 shows that the maximum principal stress for the adhesive 'Betamate 1493' is larger than for the adhesive 'Betamate $73455^{\prime}$ ' for particles of the same material, namely silica particles, which may be explained by the fact that these particles are smaller in the adhesive 'Betamate 1493', see e.g. Huber et al. [32]. Now, owing to the high elastic stiffness of the silica particles compared to the epoxy matrix, the true local critical stress in the particle corresponding to the attainment of the average value of $\sigma_{c}$ could be two to three times larger, see for example Tekoglu and Pardoen [33], and indeed a value of the fracture stress of 200 to $400 \mathrm{MPa}$ for a silica particle is a reasonable value. Further, Table 8 shows that the maximum principal stress for the adhesive 'ESP 110' is larger than for the adhesive 'Betamate 1493' for particles of a different material, namely aluminium-flake particles. This observation may be explained by the fact that the aluminium-flake particles have a higher adhesion to the epoxy matrix or contain less defects or are more ductile. They would, therefore, require a higher value of the macroscopic stress to fracture or debond. 


\section{Comparison between experimental data and numerical predictions}

All the simulations were now re-run, but this time by iteratively modifying the value of $a$ (in the wedge-peel test simulations) or the wedge thickness (in the equivalent wedge-peel test simulations representing the TDCB tests) until the failure criterion was satisfied, that is until the predicted value of the maximum principal stress at a distance ahead of the crack tip equal to $r_{c}$ was equal to $\sigma_{c}$; the average values of $r_{c}$ and $\sigma_{c}$ being employed as given in Table 8. The values of the corresponding residual radius, $R_{a}$ or $R_{2}$, of curvature for the EPFM wedge-peel test and the values of $G_{a}$ for both the EPFM wedge-peel and the LEFM TDCB test configurations were then deduced from these modelling simulations. The idea behind this exercise was to evaluate the discrepancy between the numerical predictions and experimental data and, hence, assess the accuracy of the model when used with values of the material parameters which were held constant for a given adhesive, i.e. a single pair of constant values of $r_{c}$ and $\sigma_{c}$ for any given adhesive.

\subsection{Adhesive 'Betamate 73455'}

Figure 11(a) shows a comparison between the experimental values of the average radius of curvature that were measured from the EPFM wedge-peel test specimens made using the adhesive 'Betamate $73455^{\prime}$ and the numerical predictions obtained with the model when forcing the crack to propagate along the centreline of the adhesive layer. The overall agreement is very good. Indeed, taking into account the experimental scatter, all the numerical predictions are within $\pm 10 \%$ of the experimental values. Further, the experimental decrease of the radius of curvature as a function of $h_{\text {adh }}$ was accurately captured, see Figure (11)a. The only shortcoming of the numerical results is that the radius of curvature is a somewhat underestimated (or, equivalently, the value of $G_{a}$ is overestimated) for the cases with $h_{a d h}=0.18 \mathrm{~mm}$ and $h_{a d h}=0.24 \mathrm{~mm}$. These observations could be attributed, as will be shown later for the adhesive 'Betamate 1493', to the fact that the crack was forced to run exactly along the centreline of the specimens in the simulations, whereas it always propagated a little way from the centreline in the actual experiments.

Figure 11(b) shows a comparison between the experimental values of $G_{a}$ measured using the LEFM TDCB specimens for the adhesive 'Betamate 73455 '. The numerical predictions were obtained with the model when forcing the crack to propagate along the centreline of the specimens. The overall agreement between the simulations and the experimental results is again very good. Taking into account the experimental scatter, all the predictions are within $\pm 10 \%$ of the experimental values. Further, the general trend that is observed experimentally for the values of $G_{a}$ as a function of $h_{a d h}$ is reproduced numerically. The only shortcoming of the numerical results is that, unlike the wedgepeel test results, the model now underestimates the value of $G_{a}$ for the larger values of $h_{a d h}$, and even seems to predict a steady decrease of the value of $G_{a}$ for $h_{a d h} \geq 0.4 \mathrm{~mm}$ which was not observed in the experiments. Figure 11(b) also shows the predicted values of $G_{a}$ that were obtained from the numerical simulations of the EPFM wedge-peel test in Figure 11(a). Although there are no experimental data for comparison purposes, since the complexity of the wedge-peel test makes it difficult to get an experimental value for $G_{a}$ through the use of an accurate analytical expression, the predicted values of $G_{a}$ for the EPFM wedge-peel test configuration may be directly compared to the numerically predicted, and measured values, of $G_{a}$ for the LEFM TDCB test. The values are in very 
good agreement where they overlap at $h_{a d h}=0.24 \mathrm{~mm}$, and all the predictions indicate a significant decrease of the value of $G_{a}$ with a decreasing $h_{a d h}$. This latter observation is in very good agreement with the experimental data commonly found in the literature, see, for example, Kinloch and Shaw [34] and Chai [35].

It can be concluded from the above results that the proposed model is suitable for predicting the steady-state failure of adhesive joints made using the adhesive 'Betamate 73455 ' with $h_{\text {adh }}$ values ranging from 0.1 to $1 \mathrm{~mm}$ with constant values of the material parameters $r_{c}$ and $\sigma_{c}$ of $18 \mu \mathrm{m}$ and 98 $\mathrm{MPa}$, respectively, see Table 8 .

\subsection{Adhesive 'ESP 110'}

Figure 12(a) shows a comparison between the experimental values of the radius of curvature that were measured for the adherend closer to the crack plane of the EPFM wedge-peel test specimens, made using the adhesive 'ESP 110', and the numerical predictions obtained with the model when forcing the crack to run $25 \mu \mathrm{m}$ below one of the adhesive/adherend interfaces, and evaluating the radius of curvature on the free surface of the closer adherend to that crack plane. The overall agreement between the experimental results and the theoretical predictions is excellent. Indeed, all the numerical predictions based upon the critical stress/distance model lie within the experimental error bars.

Figure 12(b) shows a comparison between the experimental values of $G_{a}$ that were measured using the LEFM TDCB test specimens made using the adhesive 'ESP 110' and the predictions obtained with the model when forcing the crack to run along the centreline of the specimens, this being the failure path observed for these joints. The overall agreement is again excellent: all numerical predictions lie within the experimental error bars. Figure 12(b) also shows the predicted values of the adhesive fracture energy that were obtained from the simulations of the EPFM wedge-peel test in Figure 12(a). For an adhesive layer thickness of $h_{a d h}=0.25 \mathrm{~mm}$, these values agree well with both the numerical predictions and the experimental data from the LEFM TDCB tests. For an adhesive layer of $h_{a d h}=0.40 \mathrm{~mm}$, the predicted value of $G_{a}$ for the wedge-peel test specimens is significantly lower than the one obtained either experimentally or numerically with the TDCB test specimens. The reason for this is that the crack was now propagated close to one of the adhesive/adherend interfaces in the simulation of the wedge-peel test specimens, unlike that observed experimentally for the TDCB test specimens where the crack runs close to the centre of the adhesive layer. To support this suggestion for the observed discrepancy, it should be noted that Kawashita et al. [27] have shown experimentally, for this same adhesive, that the former locus of joint failure does indeed result in a lower value of $G_{a}$ that due to the geometrical constraint on the plastic zone extension. It is also noteworthy that this effect is more prominent at larger values of $h_{a d h}$, as shown both experimentally and theoretically for the adhesive 'Betamate $73455^{\prime}$ by Martiny et al. [3]. This discussion further supports the idea, already suggested in Section 3.2, of developing the model in the future so as to add the capability of predicting the locus of joint failure.

The above results clearly establish that the proposed model is very suitable for predicting the steadystate failure of adhesive joints made using the adhesive 'ESP 110', with values of $h_{a d h}$ ranging from 0.1 to $1 \mathrm{~mm}$ with constant values of the material parameters $r_{c}$ and $\sigma_{c}$ of $49 \mu \mathrm{m}$ and $210 \mathrm{MPa}$, respectively, see Table 8 . 


\subsection{Adhesive 'Betamate 1493'}

Figure 13(a) shows a comparison between the experimental values of the average radius of curvature that were measured for the EPFM wedge-peel test specimens made using the adhesive 'Betamate 1493' and the numerical predictions obtained with the model when forcing the crack to run along the centreline of the specimens. For $h_{a d h}=0.05 \mathrm{~mm}$, the agreement is excellent. However, for $h_{\text {adh }}=0.18 \mathrm{~mm}$, the predicted value is only about $50 \%$ of the corresponding experimental value. Thus, as for the adhesive 'Betamate 73455 ', see Section 4.1 , the numerical model tends to underestimate the radius of curvature (or, equivalently, the value of $G_{a}$ is overestimated) for relatively thick adhesive layers. As already suggested above, the explanation for this observation is that the crack was forced to propagate along the centreline of the specimens in the simulations, whereas in the experiments it always ran a little away from the centreline of the adhesive layer. To verify this explanation, a simulation was run again, for $h_{a d h}=0.18 \mathrm{~mm}$, but now forcing the crack to propagate closer to one of the adhesive/adherend interfaces, namely at a distance of $15 \mu \mathrm{m}$ below an adhesive/adherend interface. The resulting value of the average residual radius of curvature is represented in Figure 13(a). As can be seen, the experimental value now lies between the two numerical predictions. These observations support the above explanation, since the proposed model will obviously be able to reproduce very accurately the experimental measurements by forcing the crack to run somewhere between the centreline of the specimen and a distance of $15 \mu \mathrm{m}$ from one of the adhesive/adherend interfaces. Thus, it should be noted that a significant amount of energy is dissipated through plasticity in the adhesive and the value of $G_{a}$ is therefore very sensitive, as will be shown later, to the amount of material that is made available for plastic dissipation between the crack plane and the adhesive/adherend interfaces, and the distance of the crack plane relative to the two adherends.

Figure 13(b) shows a comparison between the experimental values of $G_{a}$ that were measured using the LEFM TDCB test specimens, made using the adhesive 'Betamate 1493,' and the numerical predictions obtained with the model when forcing the crack to propagate along the centreline of the adhesive layer. The overall agreement is excellent: all the numerical predictions lie within the experimental error bars. Figure 13(b) also shows the predicted values of $G_{a}$ that were obtained from the simulations of the wedge-peel test in Figure 13(a). Although there are no experimental data that they can directly be compared with, these numerical values do agree with those obtained by modelling the TDCB test specimens, as well as with the experimental results from the TDCB test. For example, the values of $G_{a}$ from the two very different test configurations agree very well with each other at an adhesive layer thickness of $h_{a d h}=0.20 \mathrm{~mm}$, where values of $G_{a}$ from both tests are available. Further, the results from both test configurations predict the well established decrease of the value of $G_{a}$ with a decrease in $h_{a d h}$ for relatively low values of $h_{a d h}$.

The above results justify that the proposed model is able to predict the steady-state failure of adhesive joints made using the adhesive 'Betamate 1493 ' with values of $h_{a d h}$ ranging from 0.1 to 1 $\mathrm{mm}$ with constant values of the material parameters $r_{c}$ and $\sigma_{c}$ of $6.8 \mu \mathrm{m}$ and $141 \mathrm{MPa}$, respectively, see Table 8. 


\section{Parametric study}

The purpose of the present Section is to conduct a parametric study on the relationship between the values of $G_{a}$ with respect to $h_{a d h}$ that may be predicted by the critical stress/distance model as a function of many different parameters. To limit the scope, this study was concerned with (i) only the LEFM TDCB test, since this is an ISO Standard test method, (ii) only using Equation (14) for describing the strain-hardening law for the adhesive, since this equation only involves one parameter, and (iii) only critical stress/distance material parameters ranging over the values found for the three adhesives considered in the present study, since these adhesives do represent a very wide range of epoxy-based structural adhesives. To extend the applicability of this parametric study, the results were made non-dimensional.

\subsection{Dimensional analysis}

Within the context described above, a total of eleven parameters define a particular model of the TDCB test configuration. These eleven quantities consist of: (i) three parameters which define the geometry of the test (i.e. the taper parameter, $m$, the crack length, $a$, and the thickness of the adhesive layer, $h_{a d h}$ ), (ii) two parameters which describe the material behaviour of the adherends, which is assumed to be linear elastic (i.e. the Young's modulus, $E_{s}$, and the Poisson's ratio, $v_{s}$ ), (iii) four parameters which describe the elastic-plastic behaviour of the adhesive which is modelled according to Equation (14), assuming von Mises plasticity and isotropic hardening (i.e. the Young's modulus, $E$, the Poisson's ratio, $v$, the flow stress, $\sigma_{0}$, and the strain-hardening exponent, $n$ ), and, (iv) the two parameters which form the failure criterion (i.e. the maximum principal stress, $\sigma_{c}$, and the critical distance, $r_{c}$ ).

In the general case, the value of $G_{a}$ is, with the present model, a function of these eleven parameters:

$$
G_{a}=f\left(m, a, h_{a d h}, E_{s}, v_{s}, E, v, \sigma_{0}, n, \sigma_{c}, r_{c}\right)
$$

Equation (16) can be made non-dimensional by normalizing the term $h_{a d h}$ by $r_{c}$, the only length scale that is intrinsic to the adhesive in the context of the adhesive of the present model, and by normalizing the Young's modulus of the adhesive by the Young's modulus of the adherends:

$$
\frac{G_{a}}{G_{a}^{r e f}}=g\left(a \cdot m, \frac{h_{a d h}}{r_{c}}, v_{s}, \frac{E}{E_{s}}, v, \frac{\sigma_{0}}{E}, n, \frac{\sigma_{c}}{\sigma_{0}}, r_{c} \cdot m\right)
$$

The value of $G_{a}$ is normalized with respect to $G_{a}^{r e f}$, which is a 'reference value' of the adhesive fracture energy, deduced simply for the purpose of normalizing the results, and which may be obtained from the near-tip fields calculated by Irwin [36], by:

$$
G_{a}^{r e f}=\frac{1-v^{2}}{E} 2 \pi r_{c} \sigma_{0}^{2}
$$

In the Sections below the value of $G_{a} / G_{a}^{r e f}$ as a function of all the dimensionless groups appearing in the right-hand side of Equation (17) are studied; with the exception of (i) $(a \cdot m)$ and $v_{s}$, since they describe the details of the conditions of the test, whilst the focus of the present study is concerned with the adhesive, and (ii) $v$, since most epoxy adhesives have very similar values of Poisson's ratio. For comparison purposes with the results of Section 4, Table 9 shows the values of $\sigma_{0}$ and $n$ obtained 
for the three adhesives when fitting the stress versus strain curves using Equation (14), instead of Equation (15) as in Section 4, as well as stating the corresponding values of $\sigma_{c} / \sigma_{0}$ and $G_{a}^{r e f}$.

\subsection{Effect of the thickness of the adhesive layer}

Figure 14 shows the change of the normalized adhesive fracture energy, $G_{a} / G_{a}^{r e f}$, as a function of the normalized thickness, $h_{a d h} / r_{c}$, of the adhesive layer for a particular representative case. As the value of $h_{a d h}$ increases, the value of $G_{a}$ first increases, reaches a maximum and then decreases to a more or less constant plateau, a trend that is commonly observed experimentally as reported by, for example, Kinloch and Shaw [34] and Chai [35]. To gain a better understanding of this relationship, and because it is expected that most of the contribution to the value of $G_{a}$ comes from the plastic dissipation in the adhesive, $\Gamma_{p}$, the spatial distributions of the plastic dissipation in the adhesive, $\partial^{2} \Gamma_{p} / \partial X_{1} \partial X_{2}$, for the points labelled from (a) to (f) in Figure 14 were ascertained (Martiny et al. [3]) and are plotted in Figure 15. As can be seen in Figure 15, three different zones of plastic dissipation already identified by Martiny et al. [3], but in the context of a CZM approach, generally develop in the adhesive. From Figure 15(e), these are zone A, which represents the crack tip plasticity, zone B, which is due to a phenomenon referred to as reverse plastic loading, and zone $C$, which can be attributed to the shear stresses which act at the adhesive/adherend interface resulting from the restraining effect of the adherend and the through-the-thickness gradient of the $E_{11}$ strains, see Martiny et al. [3]. As $h_{a d h}$ is increased from Figure 15(a) to (c), the pattern of plastic dissipation essentially scales, and the corresponding value of $G_{a}$ increases linearly as can be seen in Figure 14 . For values of $h_{a d h}$ above values of about $10 r_{c}$, i.e. from Figure 15 (d) to (f), the patterns of plastic dissipation in zones $A$ and $B$ tend to stabilise, whilst the plastic dissipation in zone $C$ progressively vanishes so that the corresponding $G_{a}$ reaches a maximum and then decreases to a plateau value once there is no more plastic dissipation in zone $C$, as shown in Figure 14.

A more quantitative description of this evolution can be obtained by evaluating the approximate plastic dissipation in zones $A, B$ and $C$, respectively denoted by $\Gamma_{p}^{A}, \Gamma_{p}^{B}$ and $\Gamma_{p}^{C}$, following the methodology suggested in Martiny et al. [3], and plotting the evolution of these quantities with the value of $h_{a d h}$, see Figure 16 . The results in Figure 16 clearly confirm that the plastic dissipation in zones $\mathrm{A}$ and $\mathrm{B}$ initially monotonically increases with the $h_{a d h}$, since more material is available for plastic dissipation. The plastic dissipation in zones $A$ and $B$ eventually reaches a plateau value. Whereas the plastic dissipation in zone $C$ after first increasing, since there is more material available for plastic dissipation, then rapidly disappears after reaching a peak value since the crack tip has moved too far away from the adhesive/adherend interface to develop significant shearing and plastic dissipation at this location.

By subtracting an approximate value of the plastic dissipation in zone $C, \Gamma_{p}^{C}$ (as shown in Figure 16), from the value of $G_{a}$ (as shown in Figure 14), it can be quantitatively verified that zone $C$ is the origin of the peak observed in the change of $G_{a} / G_{a}^{r e f}$ with $h_{a d h} / r_{c}$, see Figure 14. It can also be seen in Figure 16 that the plastic dissipation in zones $\mathrm{A}$ and $\mathrm{B}$ reach their plateau values when $h_{a d h}$ is sufficiently large for the full height of the plastic zone under small-scale yielding conditions to develop on both sides of the crack plane, i.e. when:

$$
h_{a d h} \sim 2 r_{Y}
$$

with, from Kinloch and Shaw [34]: 


$$
r_{Y}=\frac{1}{3 \pi} \frac{E G_{a}^{S S Y}}{\left(1-v^{2}\right) \sigma_{0}^{2}}
$$

so that, together with Equation (18), Equation (19) becomes:

$$
\frac{h_{a d h}}{r_{c}} \sim \frac{2 r_{Y}}{r_{c}}=\frac{4}{3} \frac{G_{a}^{S S Y}}{G_{a}^{r e f}}
$$

where $G_{a}^{S S Y}$ is the value of $G_{a}$ under small-scale yielding conditions that was taken, as an approximation, to be equal to the plateau value of $G_{a}\left(h_{a d h} / r_{c}=65\right)$ in Figure 14.

\subsection{Effect of the critical value of the maximum principal stress}

Figure 17 shows the evolution of the normalized adhesive fracture energy, $G_{a} / G_{a}^{r e f}$, as a function of the normalized thickness, $h_{a d h} / r_{c}$, of the adhesive layer for different values of $\sigma_{c} / \sigma_{0}$ equal to 2.23, 3.43, 3.59 and 3.66. This final value has been chosen for comparative purposes to be the same as that used in Section 5.2, whereas the other values of $\sigma_{c} / \sigma_{0}$ were chosen to produce the same degree of offset between the successive curves at an adhesive layer thickness $h_{a d h} / r_{c}=65$. Figure 17 also shows the locations on these curves where $h_{a d h}=2 r_{Y}$ and the energy values that are obtained by subtracting the plastic dissipation, $\Gamma_{p}^{C}$, in zone $C$ from the adhesive fracture energy, $G_{a}$. This result further verifies the suggestion that the plastic dissipation zone $\mathrm{C}$ is the origin of the peak in the $G_{a}$ versus $h_{a d h}$ curves. As the value of $\sigma_{c} / \sigma_{0}$ is increased, the level of the stresses ahead of the crack tip increases and, as a consequence, (i) the value of $G_{a}$ is higher, (ii) the effect of zone $C$, i.e. the intensity of the peak, increases since the strain-gradient through the thickness of the adhesive layer responsible for the shearing at the adhesive/adherend interface becomes larger, and, (iii) the peak (if present) and plateau values of the $G_{a}$ occur at relatively higher values of $h_{a d h}$. Consequently, these results clearly demonstrate that not all adhesives will show a peak in the value of $G_{a}$ as a function of $h_{a d h}$; more particularly, those showing only limited plastic dissipation will not exhibit a peak. The value of $h_{a d h}$ at which $G_{a}$ reaches a plateau also correlates quite well with the predictions from Equation (21).

Figure 18 shows the evolution of the plateau value of the normalized $G_{a}$ term at relatively large values of $h_{a d h}$ (i.e. at values of $h_{a d h} / r_{c}=65$ ) as a function of $\sigma_{c} / \sigma_{0}$. The normalized $G_{a}$ term increases monotonically with the maximum principal stress, first linearly, when only limited plastic dissipation develops, and then exponentially starting from approximately $\sigma_{c} / \sigma_{0} \sim 3.25$. This result agrees very well with those obtained by Tvergaard and Hutchinson [37] under steady-state conditions in infinite elastic-plastic solids. However, they employed a CZM as a failure criterion, with the value of $\sigma_{c}$ corresponding to the peak stress in the CZM. This an important and interesting result which reveals the continuity of the present model compared to earlier studies based on a cohesive zone formulation (Pardoen et al. [10]; Martiny et al. [2,3]).

\subsection{Effect of the critical distance}

The different simulations that were run to produce the curves in Figure 17 were run again by successively using a value of $r_{c} \cdot m=0.03$ and 0.09 , instead of 0.06 , to match the range of values spanned by the three adhesives considered in Section 4 . The resulting values of the normalized $G_{a}$ term as a function of the normalized $h_{a d h}$ term did not show any significant effect upon the value of $r_{c} \cdot m$. This means that the geometrical details of the TDCB test specimens (i.e. the reference crack length, $a$, in the equivalent wedge-peel test model and the taper parameter $m$ ) are not of any 
significance, providing the value of $r_{c}$ remains relatively small compared to the dimensions of the adherends.

\subsection{Effect of the hardening exponent}

Figure 19 shows the evolution of the normalized $G_{a}$ term as a function of the normalized $h_{\text {adh }}$ term for different values of the strain-hardening exponent, $n$, equal to $0.05,0.1$ (as in Figure 17) and 0.2. Also, Figure 19 shows the effect of different values of $\sigma_{c} / \sigma_{0}$ which, for comparative purposes, give the same value of the $G_{a}$ at value of $h_{a d h} / r_{c}=65$, i.e. as shown in Figure 17.

Figure 19 firstly demonstrates that, as expected, as the strain-hardening exponent is increased then a higher value of $\sigma_{c} / \sigma_{0}$ is required to produce the same extent of plastic dissipation; and, hence, the same value of $G_{a}$ the at $h_{a d h} / r_{c}=65$. Figure 20 shows in more detail the change of the value of $G_{a}$, at value of $h_{a d h} / r_{c}=65$, as a function of $\sigma_{c} / \sigma_{0}$ for different values of the strain-hardening exponent. In the range below $\sigma_{c} / \sigma_{0} \sim 3$, where the value of $G_{a}$ increases linearly a function of $\sigma_{c} / \sigma_{0}$, the effect of the strain-hardening exponent is negligible, since there is only limited plastic dissipation. For values of $\sigma_{c} / \sigma_{0}$ above about 3 , the value of $G_{a}$ starts increasing exponentially at a higher values of $\sigma_{c} / \sigma_{0}$; and this increase is less steep as the strain-hardening exponent is increased, since the adhesive is now capable of accommodating higher stresses with smaller strains and less plastic dissipation. These observations are again in very good agreement with results obtained by Tvergaard and Hutchinson [37]. Returning to Figure 19, secondly, it may be seen that, as the hardening exponent is decreased for a given level of the adhesive fracture energy, the effect of zone $\mathrm{C}$, i.e. the cause of the presence of the peak toughness, increases. This arises since the strains at the crack tip need to be higher to produce the same level of $G_{a}$, whilst the restraining effect of the high-modulus adherends at the adhesive/adherend interface remains the same. This means that the through-thethickness gradient of strains and, hence, the shear stresses and strains at the adhesive/adherend are higher. Thirdly, Figure 19 demonstrates that the effect of the strain-hardening exponent is more prominent for higher values of $G_{a}$; since the higher the value of $G_{a}$ then the larger the plastic dissipation and, hence, the larger the differences between the different values of the strainhardening exponent.

\subsection{Effect of the Young's modulus of the adhesive}

Figure 21 shows the evolution of the normalized $G_{a}$ term as a function of the normalized $h_{\text {adh }}$ term for different values of the normalized Young's modulus of the adhesive, $E / E_{S}$, equal to $0.04,0.06$ (as in Figure 17) and 0.08 , and, for each of these, for four different values of $\sigma_{c} / \sigma_{0}$, which are the same as those used in Figure 17. It may be observed that the normalized $G_{a}$ term is relatively independent of the value of $E / E_{s}$ at large thicknesses of the adhesive layer (i.e. $h_{a d h} / r_{c}=65$ ). This means that that $G_{a}$ is measured experimentally for relatively thick adhesive layers is largely independent of the Young's modulus of the adherends, which supports the idea that the LEFM TDCB test gives a value for $G_{a}$, that is characteristic of the adhesive material when failure through the adhesive layer is observed, albeit a function of the selected value of $h_{a d h}$ of the adhesive layer.

Further, Figure 21 shows also that, as the Young's modulus of the adhesive is decreased for a given value of $\sigma_{c} / \sigma_{0}$, the effect of plastic dissipation in zone $\mathrm{C}$ increases, since the restraining effect of the high-modulus adherends at the adhesive/adherend interface becomes more important, compared to the modulus of the adhesive. This means that the through-the-thickness gradient of the $E_{11}$ strains and the shear stresses and strain at the adhesive/adherend are larger. Finally, Figure 21 shows that 
this effect is more important for relatively very high values of $G_{a}$; since the higher the value of $G_{a}$ the larger the effect of zone $\mathrm{C}$, regardless of the value of $E / E_{S}$.

\subsection{Effect of the initial yield stress of the adhesive}

Figure 22 shows the change of the normalized $G_{a}$ term as a function of the normalized $h_{\text {adh }}$ term for different values of the normalized initial yield stress of the adhesive, $\sigma_{0} / E$, for values equal to 0.005 , 0.015 (as in Figure 17) and 0.025. Also, shown are the effects of three different values of $\sigma_{c} / \sigma_{0}$ identical to those used in Figure 17, with the exception of the largest value of 3.66 which gave, together with a value of $\sigma_{0} / E$ equal to 0.005 , far higher values of $G_{a}$ that would have been difficult to represent clearly on the same plot.

As the initial yield stress of the adhesive is decreased, (i) the overall level of the adhesive fracture energy increases since the yield strain is smaller and, hence, plastic dissipation is more easily generated, and, (ii) the effect of zone $\mathrm{C}$ is more prominent since the same shear strains at the adhesive/adherend interface induce more plastic dissipation. Figure 22 also shows that this effect is more important for relatively large values of $\sigma_{c} / \sigma_{0}$ for which the associated strains are larger. These results, as far as the curves for $\sigma_{c} / \sigma_{0}>2.23$ are concerned, are not in agreement with the results of Tvergaard and Hutchinson [37] which showed that the ratio $\sigma_{0} / E$ had a negligible effect. The reason for is that Tvergaard and Hutchinson [37] studied infinite elastic-plastic solids for which the conditions of small-scale yielding conditions applied, which is not case here. This may be demonstrated by considering the case for when the height of the plastic zone is not small compared to the value of $h_{a d h}$, for example:

$$
2 r_{Y}>\frac{1}{10} h_{a d h}
$$

or, in non-dimensional form, using the right-hand side of Equation (21) when:

$$
\frac{4}{3} \frac{G_{a}^{S S Y}}{G_{a}^{r a f}}>\frac{1}{10} \frac{h_{a d h}}{r_{c}}
$$

Noting that in Figure 22 the normalized $h_{a d h}$ term being considered ranges up to $h_{a d h} / r_{c}=65$, Equation (22) now becomes:

$$
\frac{G_{a}^{S S Y}}{G_{a}^{r e f}}>\sim 5
$$

which is indeed the case for the curves with $\sigma_{c} / \sigma_{0}>2.23$.

\subsection{Final comments}

All the results of the above parametric study demonstrate many similarities with the predictions that were obtained [3] earlier where a CZM approach was adopted. However, the CZM approach has been found not to be capable of predicting the change of $G_{a}$ as a function of $h_{a d h}$ when a wide range of thickness values are employed, see the Appendix. The origin of the problem was tentatively proposed to result from the constant value of the energy spent in the cohesive zone, which was assumed to be independent of the degree of constraint at the crack tip. A damage-based approach was the most obvious way to circumvent this issue. The present parametric study shows that indeed the use of an extremely simple damage model allows the predictions of the present model to encompass a far larger range of thicknesses of the adhesive layer than a CZM approach using the 
same number of material parameters. In the present model, a higher degree of constraint leads to the attainment of the critical stress at smaller plastic strains in the near crack-tip region. It is exactly as if the local energy spent in an analogous CZM would be smaller. For the future, more elaborate damage models could obviously be used, at the expense of more material parameters being required. The present study also suggests that the nucleation of damage might be, for many adhesive systems, the key element to model. Our view is that the most critical element to carefully address in future work is a more accurate model of the plasticity of the adhesive involving a viscoelasticviscoplastic description which is dependent upon the local hydrostatic stress.

\section{Conclusions}

A numerical model capable of predicting the mode I fracture of adhesive joints under steady-state conditions has been developed, using a critical value of the maximum principal stress, $\sigma_{c}$, at a critical distance, $r_{c}$, ahead of the crack tip as the failure criterion. The material parameters were identified for three different structural, epoxy-based adhesive systems which showed very different values of their adhesive fracture energies, $G_{a}$, spanning the range of about $200-6500 \mathrm{~J} / \mathrm{m}^{2}$. The critical stress was related to the principal stress that is needed to debond or cleave these particles, and so initiate a void or micro-crack that would cause crack propagation. The order of magnitude of the critical distances was linked, with the help of micrographs of the fracture surfaces, to the average size and/or spacing of the second-phase particles in the adhesive. The numerical predictions were in excellent agreement with the experimental data, using constant values of the material parameters, $\sigma_{c}$ and $r_{c}$, for a given adhesive. This observation was valid over a very wide range of thicknesses, $h_{a d h}$, of the adhesive layer between 0.1 and $1.0 \mathrm{~mm}$ and for two very different test configurations, namely the LEFM TDCB test and the EPFM wedge-peel test.

A detailed, non-dimensional parametric study was then conducted to study the effect of the different material parameters on the predicted relationships between the normalized adhesive fracture energy and the normalized thickness of the adhesive layer for the LEFM TDCB test. Several noteworthy points emerged. Firstly, the numerical results showed that the model was capable of reproducing an experimental trend commonly reported previously in the literature: namely that the value of $G_{a}$ first increases linearly, then passes through a peak and then decreases down to a plateau value (equivalent to the bulk adhesive value) as the value of $h_{a d h}$ is increased. The physical explanation for this trend was found to be directly linked to the development of the different regions of plastic dissipation in the adhesive layer. This proposal was confirmed by quantitatively analysing the predicted spatial distributions of these region of plastic dissipation in the adhesive. These results also indicated that not all adhesives will show a peak in the adhesive fracture energy as a function of the thickness of the adhesive layer; more particularly, those showing only limited plastic dissipation will not exhibit a peak. Secondly, the parametric study revealed that the value of $G_{a}$ was largely dependent upon: (i) the value of the critical maximum principal stress, and (ii) the yield stress and strain-hardening properties of the adhesive, i.e. the value of $G_{a}$ being magnified by relatively low values of the initial yield stress and of the strain-hardening exponent both of which promoted plastic dissipation in the adhesive layer. These observations emphasise the importance of using the complete elastic-plastic mechanical properties to model the adhesive layer in order to achieve accurate numerical predictions. Thirdly, the results of the parametric study revealed that the values of the $G_{a}$ obtained from the LEFM TDCB test were virtually independent of the geometry and the 
material used for the adherends. This strongly supports the idea that this test standard method gives a value of the adhesive fracture energy that is a 'characteristic material property' of the adhesive material, albeit a function of the selected value of the thickness, $h_{a d h}$, of the adhesive layer and, of course, assuming that the locus of joint failure is located along the centreline of the adhesive layer. Finally, the proposed model shows considerable potential in engineering applications with respect to predicting the failure properties of a wide range of adhesives, especially via the use of the results from the parametric study. 


\section{Acknowledgements}

The present work was carried out under the SINUS project, jointly funded by Wallonia (Belgium) and the European ERDF funds. The authors are grateful to Dr. L. Kawashita (formerly with Imperial College London) and Mr. M. Sinnaeve (Université catholique de Louvain) for their experimental assistance.

\section{References}

1. Kinloch AJ. Adhesives in engineering. Proc Intst Mech Engrs 1997; Part G 211: 307-35.

2. Martiny Ph, Lani F, Kinloch AJ, Pardoen, T. Numerical analysis of the energy contributions in peel tests: a steady-state multilevel finite element approach. Int J Adhes Adhes 2008; 28: 222-36.

3. Martiny Ph, Lani F, Kinloch AJ, Pardoen T. A multiscale parametric study of mode I fracture in metal-to-metal low-toughness adhesive joints. Int J Fract 2012; 173: 105-33.

4. Tvergaard V, Hutchinson, JW. Toughness of an interface along a thin ductile layer joining elastic solids. Philos Mag 1994; A70: 641-56.

5. Tvergaard V Hutchinson, JW. On the toughness of ductile adhesive joints. J Mech Phys Solids 1996; 44: 789-800.

6. Kafkalidis MS, Thouless MD, Yang QD, Ward SM. Deformation and fracture of adhesive layers constrained by plastically-deforming adherends. J Adhes Sci Technol 2000; 14: 1593-1607.

7. Yang QD, Thouless MD, Ward SM. Analysis of the symmetrical $90^{\circ}$-peel test with extensive plastic deformation. J Adhes 2000; 72: 115-32.

8. Georgiou I, Hadavania H, Ivankovic A, Kinloch AJ, Tropsa V. Williams JG. Cohesive zone models and the plastically deforming peel test. J Adhes 2003; 79: 239-65.

9. Ferracin T. Mechanics of failure in adhesively bonded steel assemblies. Université catholique de Louvain, Belgium, PhD Thesis; 2003.

10. Pardoen T, Ferracin T, Landis CM, Delannay, F. Constraint effects in adhesive joint fracture. J Mech Phys Solids 2005; 53: 1951-83.

11. Salomonsson $\mathrm{K}$, Andersson T. Modelling and parameter calibration of an adhesive layer at the meso level. Mech Mater 2008; 40:48-65.

12. Cooper V, Ivankovic A, Karac A, McAuliffe D, Murphy N. Effects of bond gap thickness on the fracture of nano-toughened epoxy adhesive joints. Polymer 2012; to be published.

13. Siegmund T, Brocks, W. Prediction of the work of separation and implications to modeling. Int J Fract 1999; 99: 97-116.

14. Pardoen T, Hachez F, Marchioni B, Blyth PH, Atkins, AG. Mode I fracture of sheet metal. J Mech Phys Solids 2004; 52: 423-52. 
15. Tvergaard V, Hutchinson JW. Effect of strain-dependent cohesive zone model on predictions of crack growth resistance. Int J Solids Struct 1996; 33: 3297-308.

16. Ritchie RO, Knott JF, Rice JR. On the relationship between tensile stress and fracture toughness in mild steels. J Mech Phys Solids 1973; 21:395-410.

17. Whitney JM, Nuismer RJ. Stress fracture criteria for laminated composites containing stressconcentrations. J Compos Mater 1974; 8:253-265.

18. Kinloch AJ, Williams JG. Crack blunting mechanisms in polymers. J Materials Sci 1980; 15: 98797.

19. Clarke JD, McGregor IJ. Ultimate tensile stress over a zone: a new failure criterion for adhesive joints. J Adhes 1993; 42: 227-45.

20. Taylor D. The theory of critical distances. Eng Fract Mechs 2008; 75: 1696-1705

21. Da Silva LFM, Adams RD. The strength of adhesively bonded T-joints. Int J Adhes Adhes 2002; 22: 311-15.

22. Blackman BRK, Hadavinia H, Kinloch AJ, Paraschi M, Williams JG. The calculation of adhesive fracture energies in mode I: revisiting the tapered double cantilever beam (TDCB) test. Eng Fract Mech; 2003; 70: 233-48.

23. Georgiou I. The fracture of adhesively-bonded aluminium joints for automotive structures. Imperial College London, United Kingdom, PhD Thesis; 2003.

24. Pardoen T, Pineau A. Failure mechanisms of metals. In: Comprehensive structural integrity encyclopedia, vol. 2., Elsevier, Amsterdam; 2007. 684-797.

25. Crocombe AD, Richardson G, Smith PA. A Unified Approach for Predicting the Strength of Cracked and Non-Cracked Adhesive Joints. J Adhes 1995; 49: 211-244.

26. Dean RH, Hutchinson JW. Quasi-static steady crack growth in small-scale yielding. ASTM STP 700, 1980; 383-405.

27. Kawashita LF, Kinloch AJ, Moore DR, Williams JG. The influence of bond line thickness and peel arm thickness on adhesive fracture toughness of rubber toughened epoxy-aluminium alloy laminates. Int J Adhes Adhes 2008; 28: 199-210.

28. Hadavinia H, Kawashita L, Kinloch AJ, Moore DR, Williams JG. A numerical analysis of the elasticplastic peel test. Eng Fract Mech 2006; 73: 2324-35.

29. Xia L, Shih CF. Ductile crack growth: I. A numerical study using computational cells with microstructurally based length scales. J Mechs Phys Solids 1995; 43 : 233-59.

30. Pardoen T, Hutchinson JW. Micromechanics-based model for trends in toughness of ductile metals. Acta Materialia 2003; 51: 133-48. 
31. Pardoen T, Doghri I, Delannay F. Experimental and numerical comparison of void growth models and coalescence criteria for the prediction of ductile fracture in copper bars. Acta Materialia 1998; 46:541-552.

32. Huber $G$, Bréchet $Y$, Pardoen T. Predictive model for void nucleation and void growth controlled ductility in quasi-eutectic cast aluminium alloys. Acta Materialia 2005; 53: 2739-49.

33. Tekoğlu C, Pardoen T. A micromechanics based damage model for composite materials. Intern J Plast 2010; 26: 549-69.

34. Kinloch AJ, Shaw SJ. The fracture resistance of a toughened epoxy adhesive. J Adhes 1981; 12: 59-77.

35. Chai H. Bond thickness effect in adhesive joints and its significance for mode I interlaminar fracture of composites. ASTM STP 893, 1986; 209-31.

36. Irwin GR. Analysis of stresses and strains near the end of crack traversing a plate. J Appl Mech 1957; 24: 361-64.

37. Tvergaard V, Hutchinson JW. The relation between crack growth resistance and fracture process parameters in elastic-plastic solids. J Mech Phys Solids 1992; 40: 1377-97. 


\section{Appendix}

Figure 23(a) shows a comparison between the experimental values of the average radius of curvature, that was measured from the EPFM wedge-peel test specimens made using the adhesive 'ESP 110', and the numerical predictions obtained by Martiny et al. [2]. The numerical predictions were ascertained from a model from Martiny et al. [2,3] where the CZM possessed zero height and had material parameters which defined the shape and size of the CZM. The local fracture process was simulated with the cohesive zone and the local energy dissipation in the adhesive, ahead of the crack front, was accounted for by embedding the CZM between layers of elastic-plastic solid elements which represent the adhesive layer. In Figure 23(a) the agreement is excellent as all the numerical values lie within the experimental error bars. However, Figure 23(b) shows a comparison between the experimental values of the adhesive fracture energy, $G_{a}$, that were measured from the LEFM TDCB test specimens using the adhesive 'ESP 110', and the numerical predictions that were obtained when using this model and the material parameter values of Martiny et al. [2]. Although the agreement is reasonably good for the range of adhesive layer thickness as used for Figure 23(a), the numerical predictions greatly underestimate the experimental values of $G_{a}$ as the thickness of the adhesive layer is increased above this range. Figure 23(b) also shows that as the energy, $\Gamma_{0}$, expended in the CZM is kept constant, the total energy expenditure in the adhesive layer, $\Gamma_{b}$ (which is added to $\Gamma_{0}$ to give the value of $G_{a}$ ) does not significantly increase as the thickness, $h_{a d h}$, of the adhesive layer is increased. Thus, the numerical predictions cannot follow the increase in $G_{a}$ that is observed experimentally.

The explanation for these observations may be found in Figure 24 which was reproduced from Tvergaard and Hutchinson [37] who calculated the steady-state toughness of elastic-plastic solids. As for the adhesive layer in Martiny et al. [2], they employed continuum elements with an embedded cohesive zone model with zero thickness. Figure 24 shows that, with the material parameters used by Martiny et al. [2] of $\hat{\sigma} / \sigma_{0} \sim 3, \Gamma_{0} \sim 800 \mathrm{~J} / \mathrm{m}^{2}$ and $n \sim 0.2$, the energy expenditure in the bulk of the adhesive, $\Gamma_{b}$, that can be expected is of the order of $0.2 \Gamma_{0} \sim 160 \mathrm{~J} / \mathrm{m}^{2}$, as observed in Figure $23(\mathrm{~b})$. In other words, for the strain-hardening exponent characterising the adhesive 'ESP 110', the value of the peak stress, $\hat{\sigma}$, that was used by Martiny et al. [2] in their CZM is not sufficiently high to generate sufficient energy dissipation in the adhesive. Thus, the increase in the adhesive fracture energy that is observed experimentally from $h_{a d h}=0.32$ to $0.88 \mathrm{~mm}$ cannot be predicted from this CZM employing the parameters used in the CZM that were used for the lower values of $h_{a d h}$.

Thus, the authors attempted to check whether, by using larger values of the peak stress, $\hat{\sigma}$, in the CZM, it was at all possible to reproduce the experimental results for $h_{a d h}=0.32$ to $0.88 \mathrm{~mm}$. They therefore let $\hat{\sigma}$ increase and adapted the value of $\Gamma_{0}$ accordingly for the CZM to reproduce exactly the experimentally measured value of the adhesive fracture energy at an adhesive layer thickness of $h_{a d h}=0.32 \mathrm{~mm}$. They then used each of these pair of values $\left(\hat{\sigma}, \Gamma_{0}\right)$ in the CZM to calculate the adhesive fracture energy at an adhesive layer thickness of $h_{a d h}=0.88 \mathrm{~mm}$. The resulting change of the adhesive fracture energy as a function of the peak stress, $\hat{\sigma}$, is shown in Figure 25 . It can be seen that it is not possible with the model suggested by Martiny et al. [2] to reproduce numerically the increase in the adhesive fracture energy that is measured experimentally, regardless of the value of $\hat{\sigma}$ selected. Indeed, as $\hat{\sigma}$ is increased, the value of $\Gamma_{b}$ does increase but the value of $\Gamma_{0}$ decreases at the same time so as to be consistent with the value of $G_{a}$ at $h_{a d h}=0.32 \mathrm{~mm}$. Hence, overall, the value of $G_{a}$ at a value of $h_{a d h}=0.88 \mathrm{~mm}$ is not significantly increased. As a final comment, this 
observation also leads to an understanding as to why the model suggested in the present paper does not suffer from the same shortcomings. Namely, it does not include any CZM and, as a consequence, does not experience the effect of any contribution to the value of the adhesive fracture energy being forced to decrease, as the value of the critical maximum principal stress is increased. 


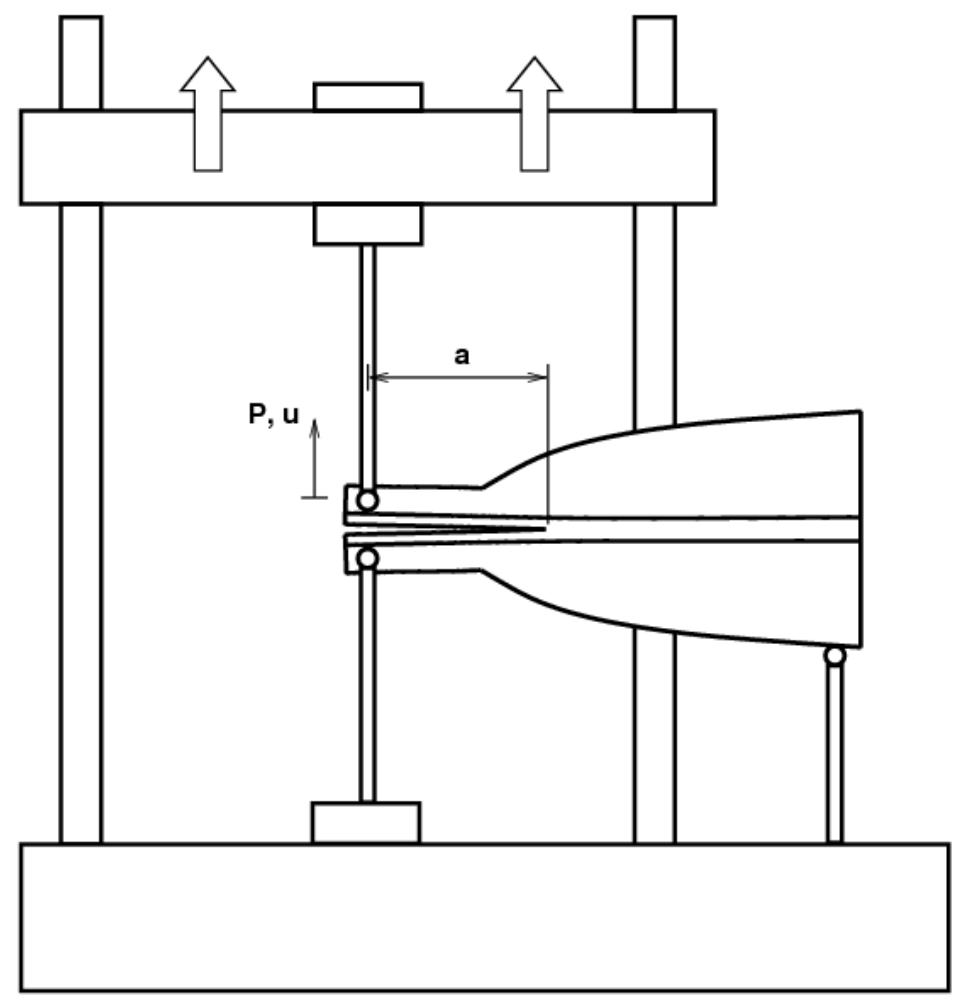

(a)

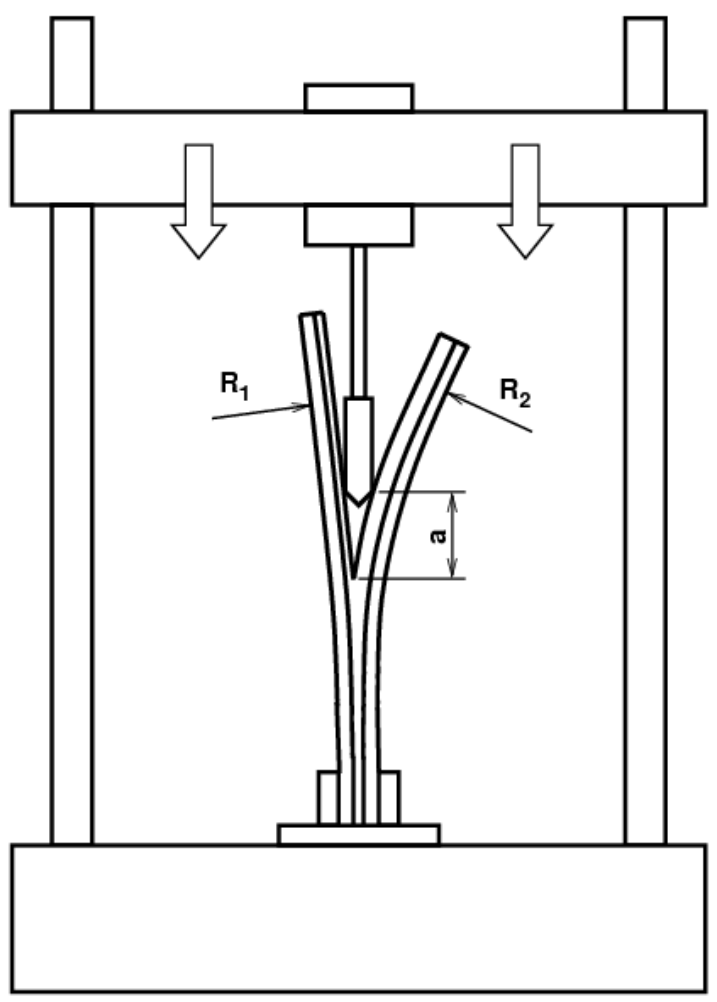

(b)

Figure 1. The experimental set-up for (a) the LEFM tapered double-cantilever beam (TDCB) test specimen, and (b) the EPFM wedge-peel test specimen 


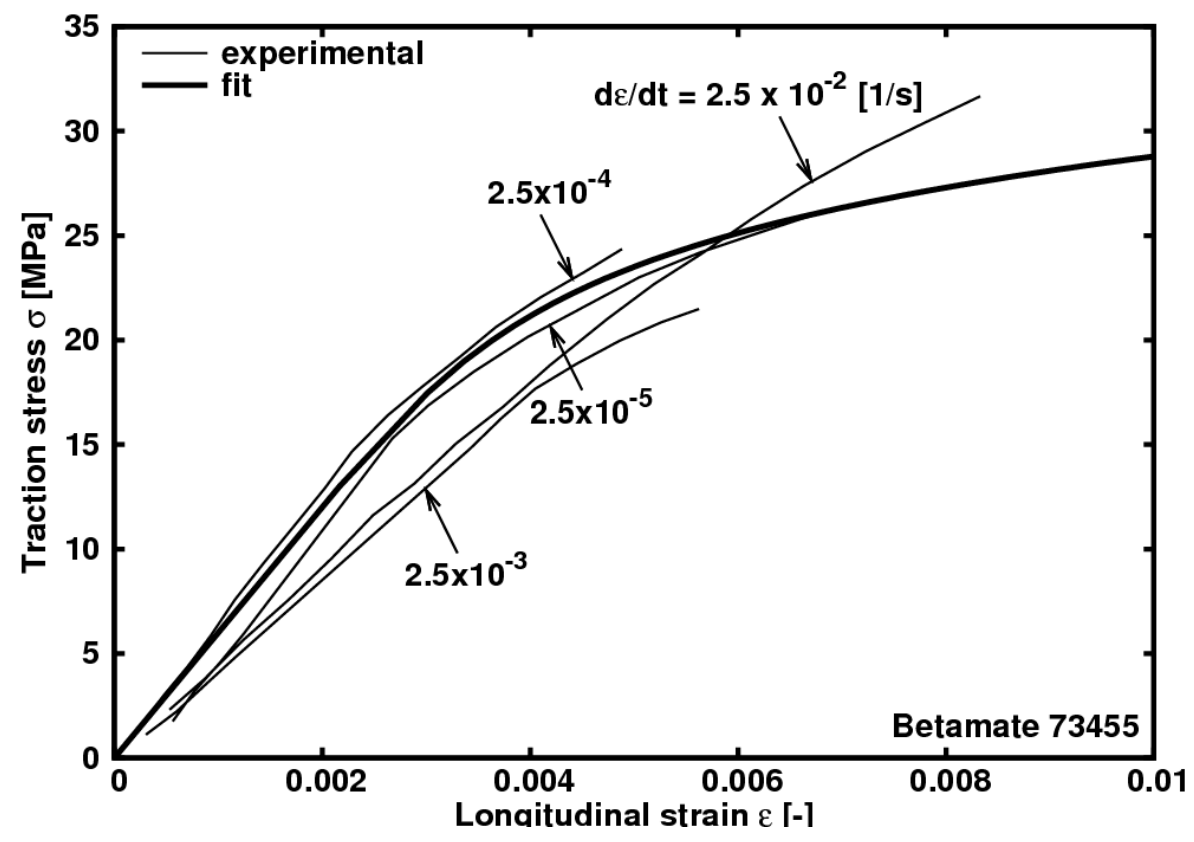

Figure 2. The experimental tensile stress versus strain curves and corresponding fitted model for the adhesive 'Betamate 73455' 


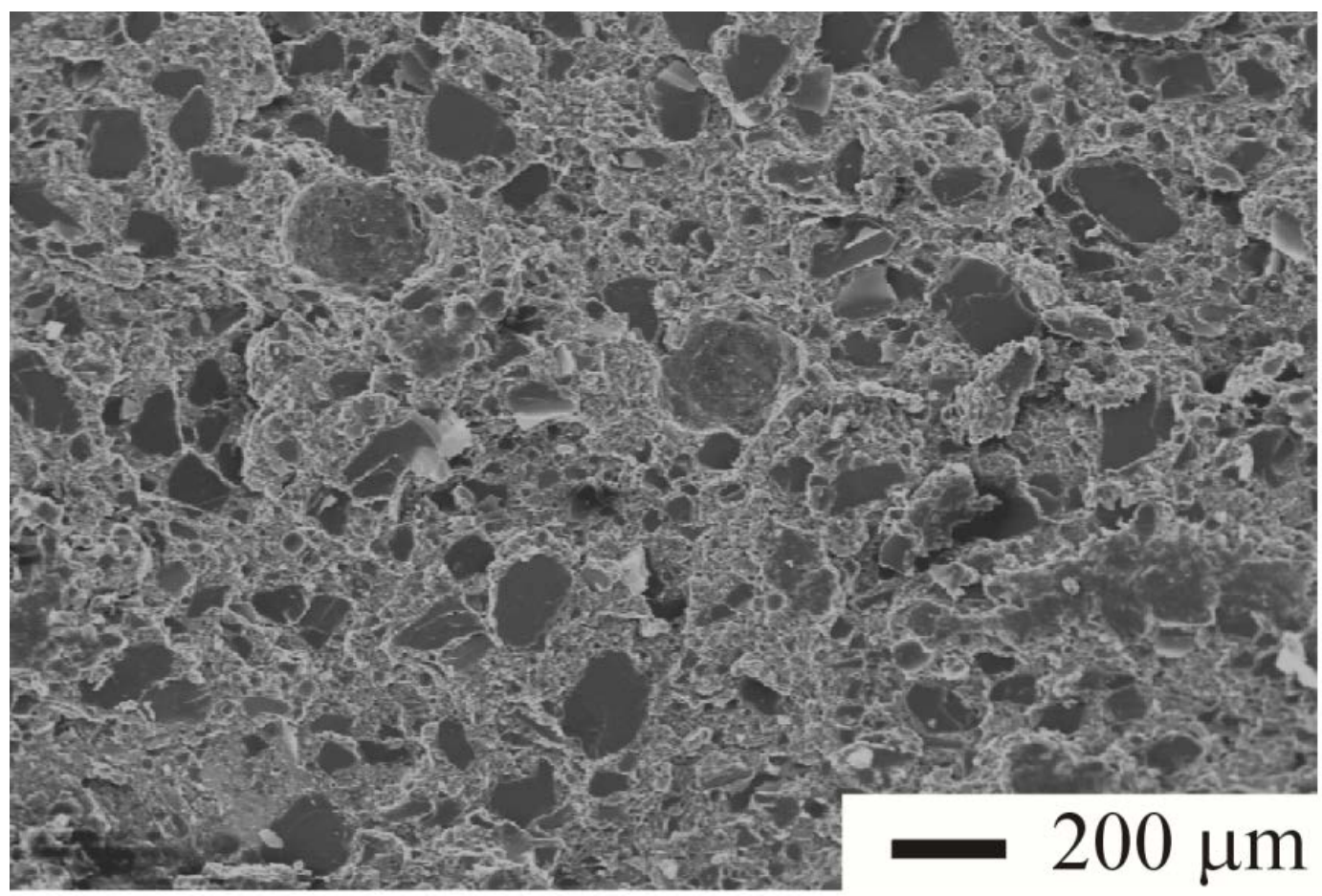

(a)

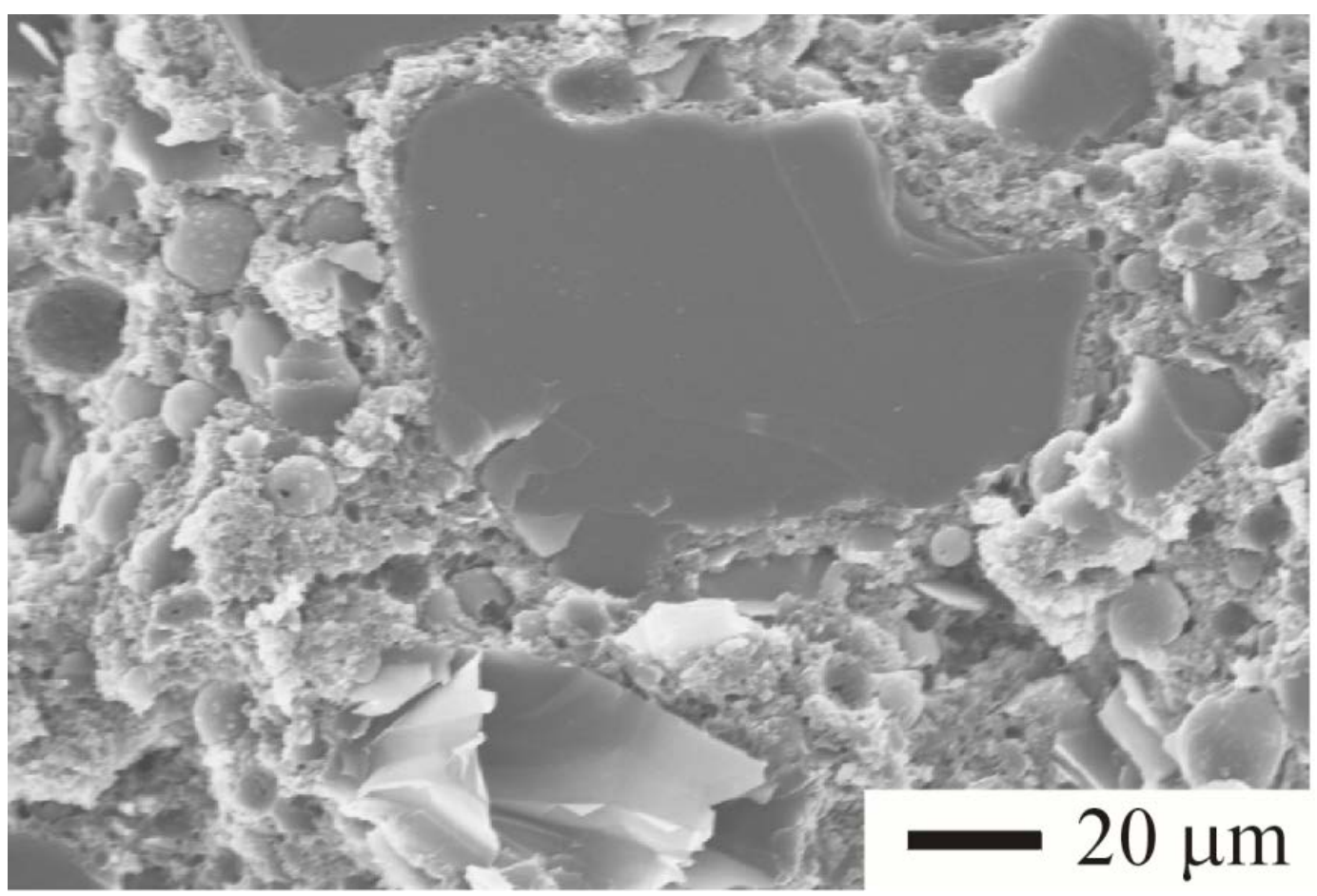

(b)

Figure 3. Typical fracture surface of the adhesive 'Betamate 73455 ' at two different magnification levels 


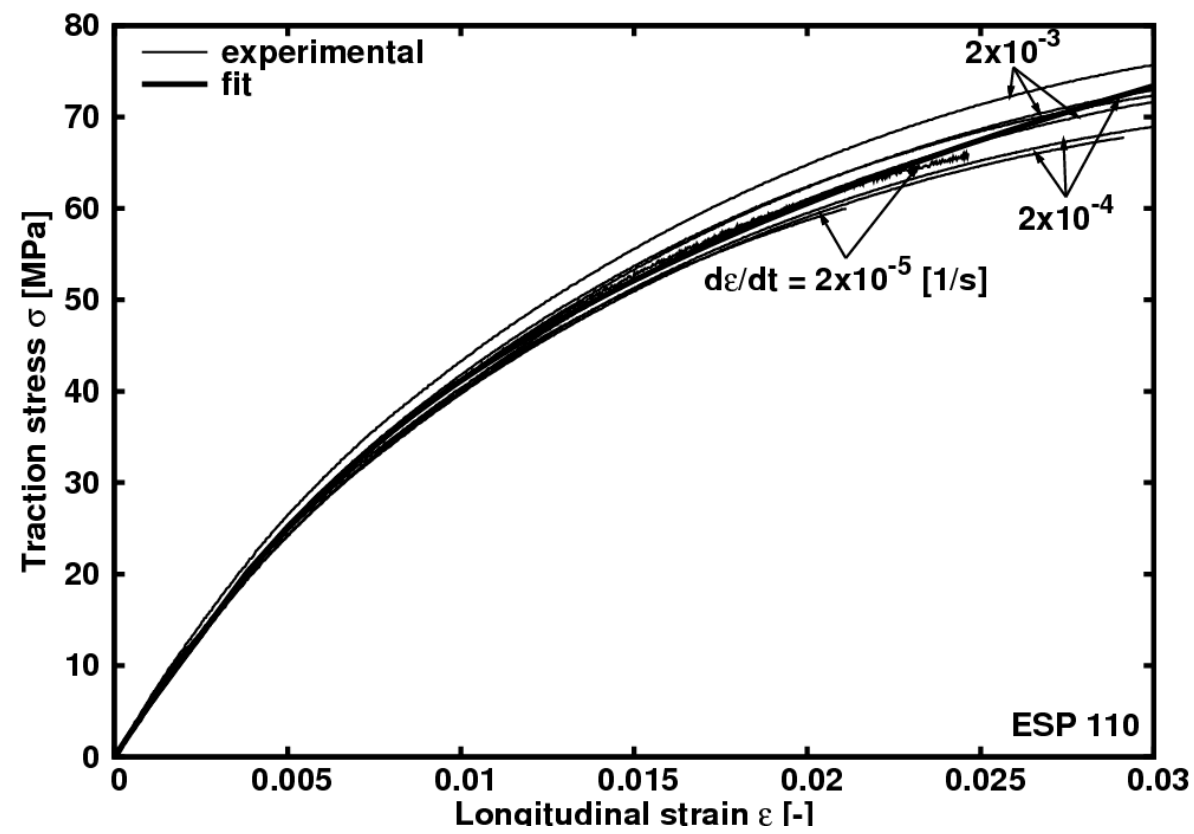

Figure 4. The experimental tensile stress versus strain curves and corresponding fitted model for the adhesive 'ESP 110' 


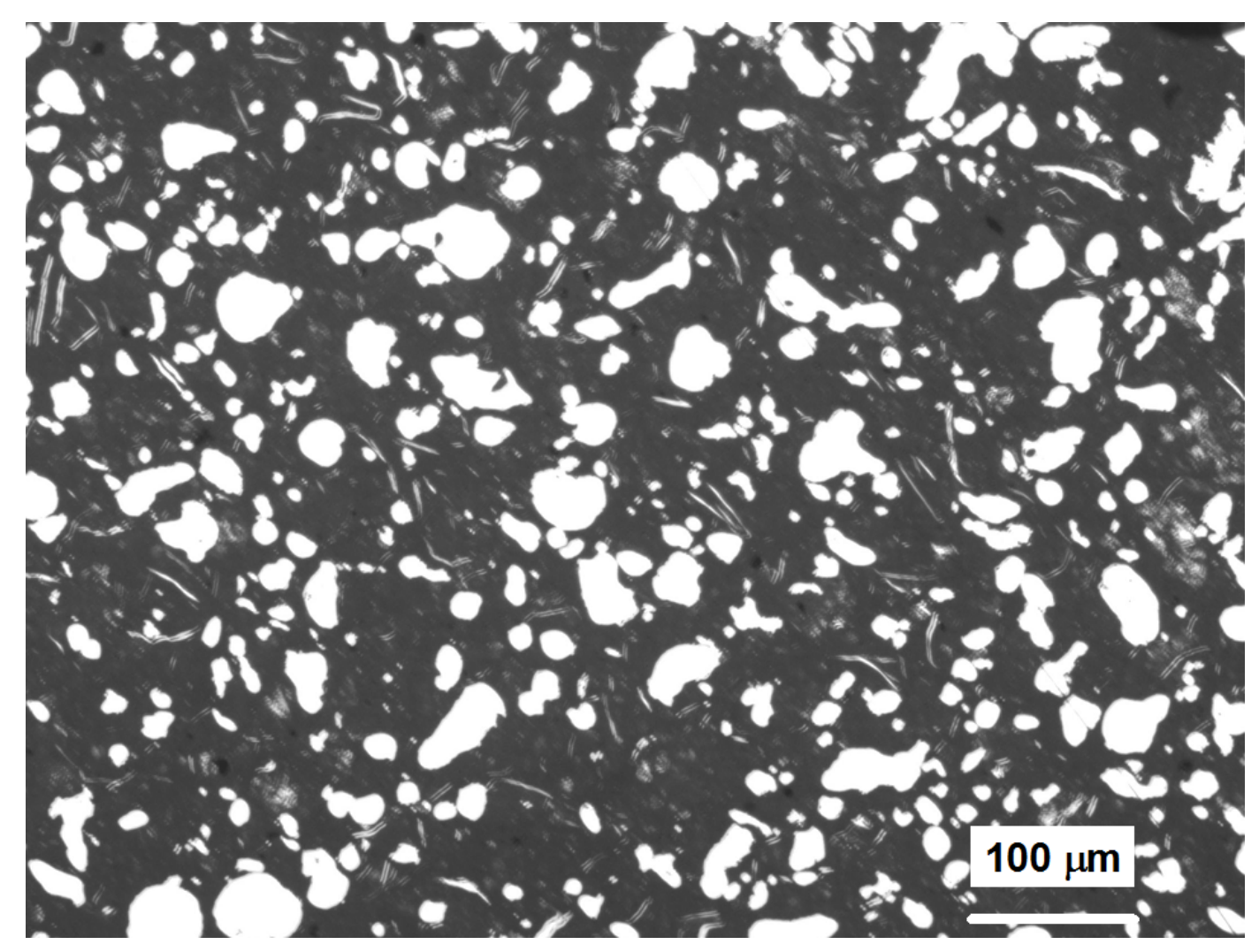

(a)

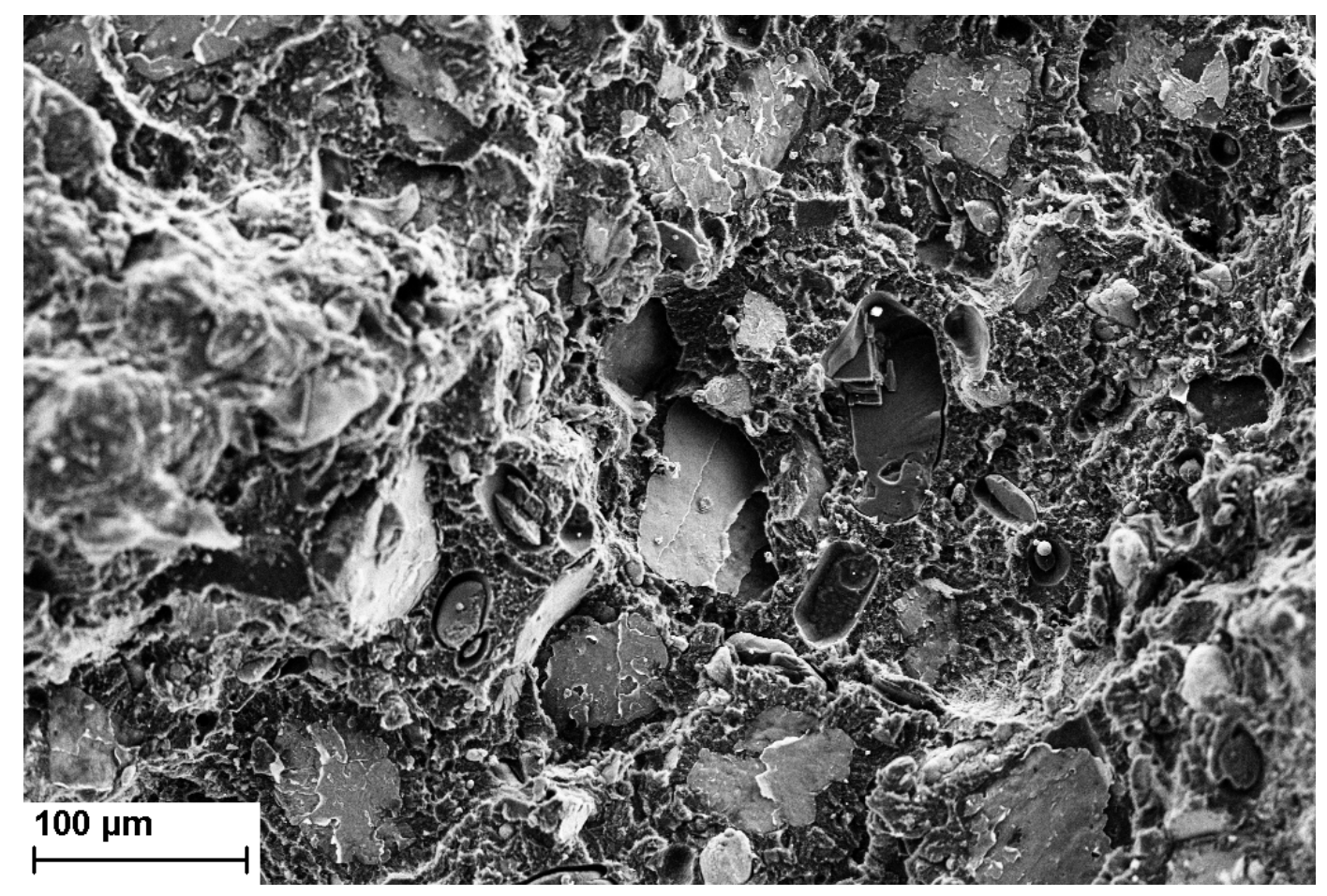

(b)

Figure 5. Micrographs of the adhesive 'ESP 110' obtained by scanning electron microscopy: (a) polished section and (b) fracture surface of a TDCB test specimen 


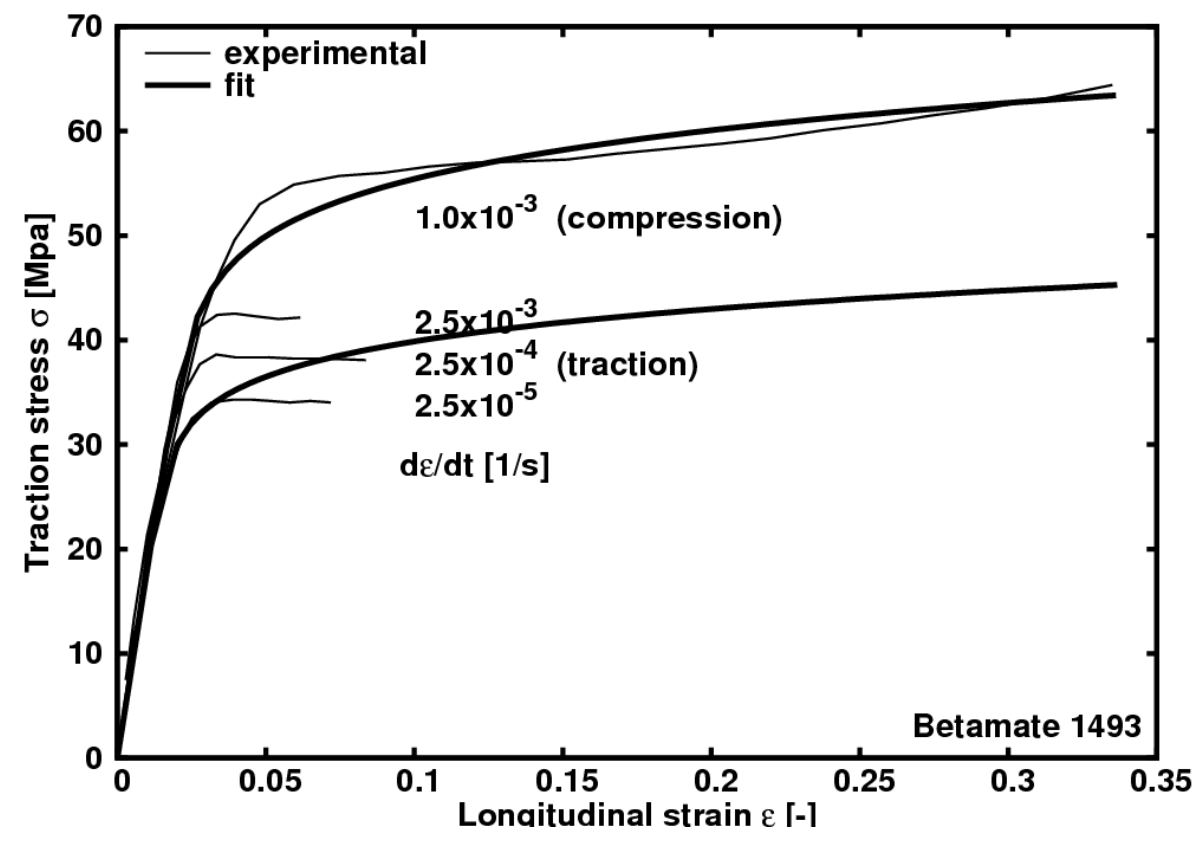

Figure 6. The experimental tensile and compression stress versus strain curves and corresponding fitted models for the adhesive 'Betamate 1493' 


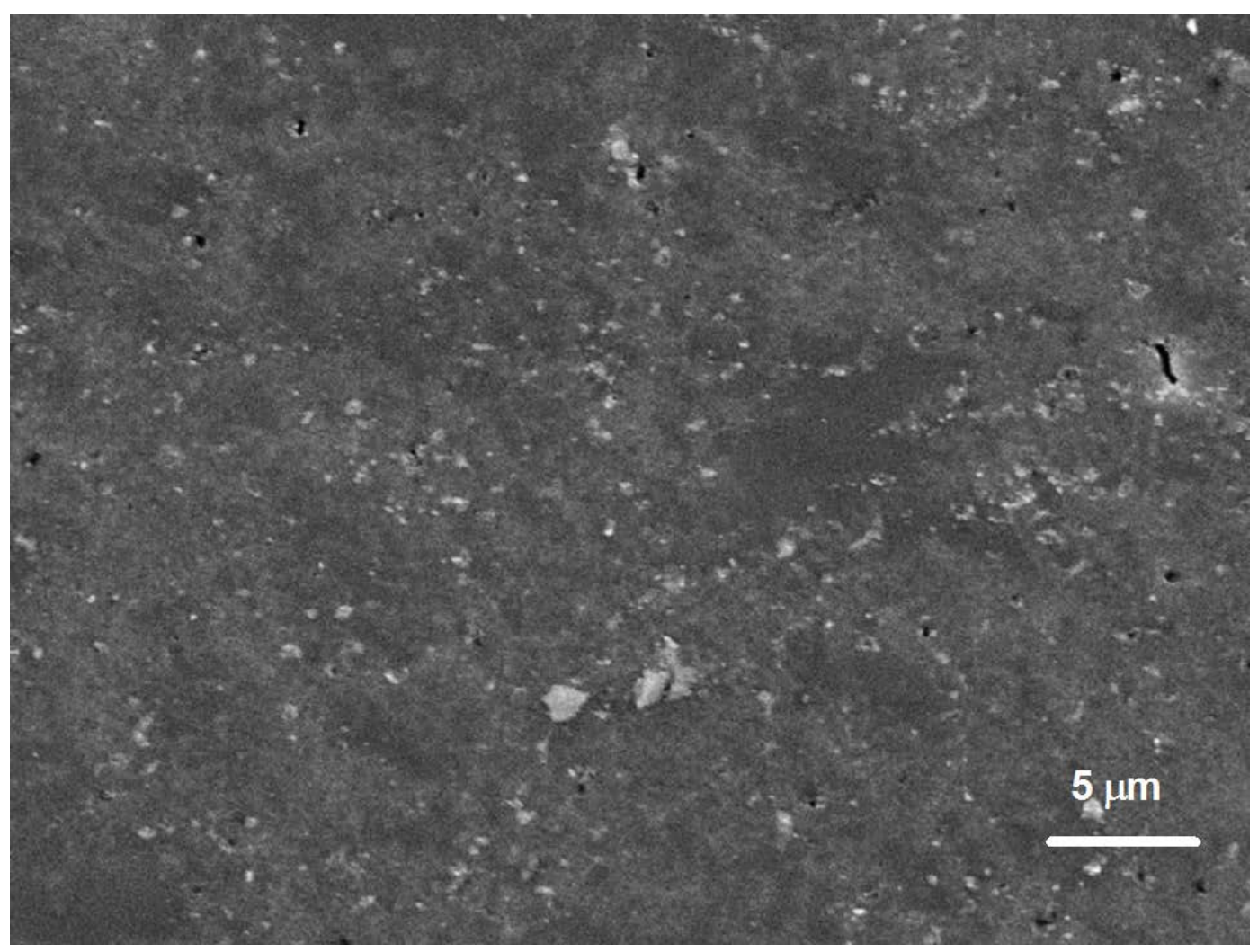

(a)

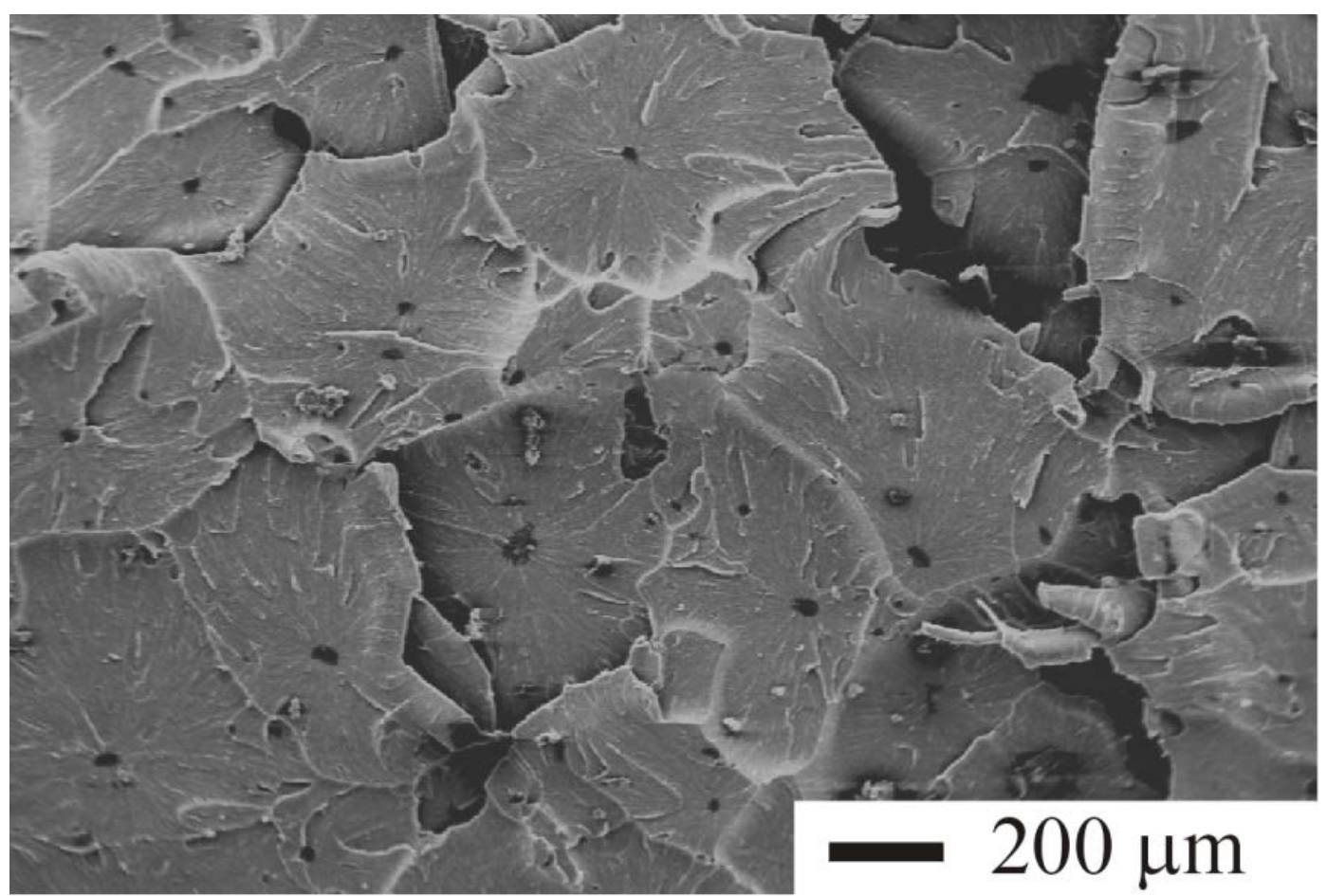

(b)

Figure 7. Micrographs of the adhesive 'Betamate 1493' obtained by scanning electron microscopy: (a) polished section and (b) fracture surface of a TDCB test specimen. 


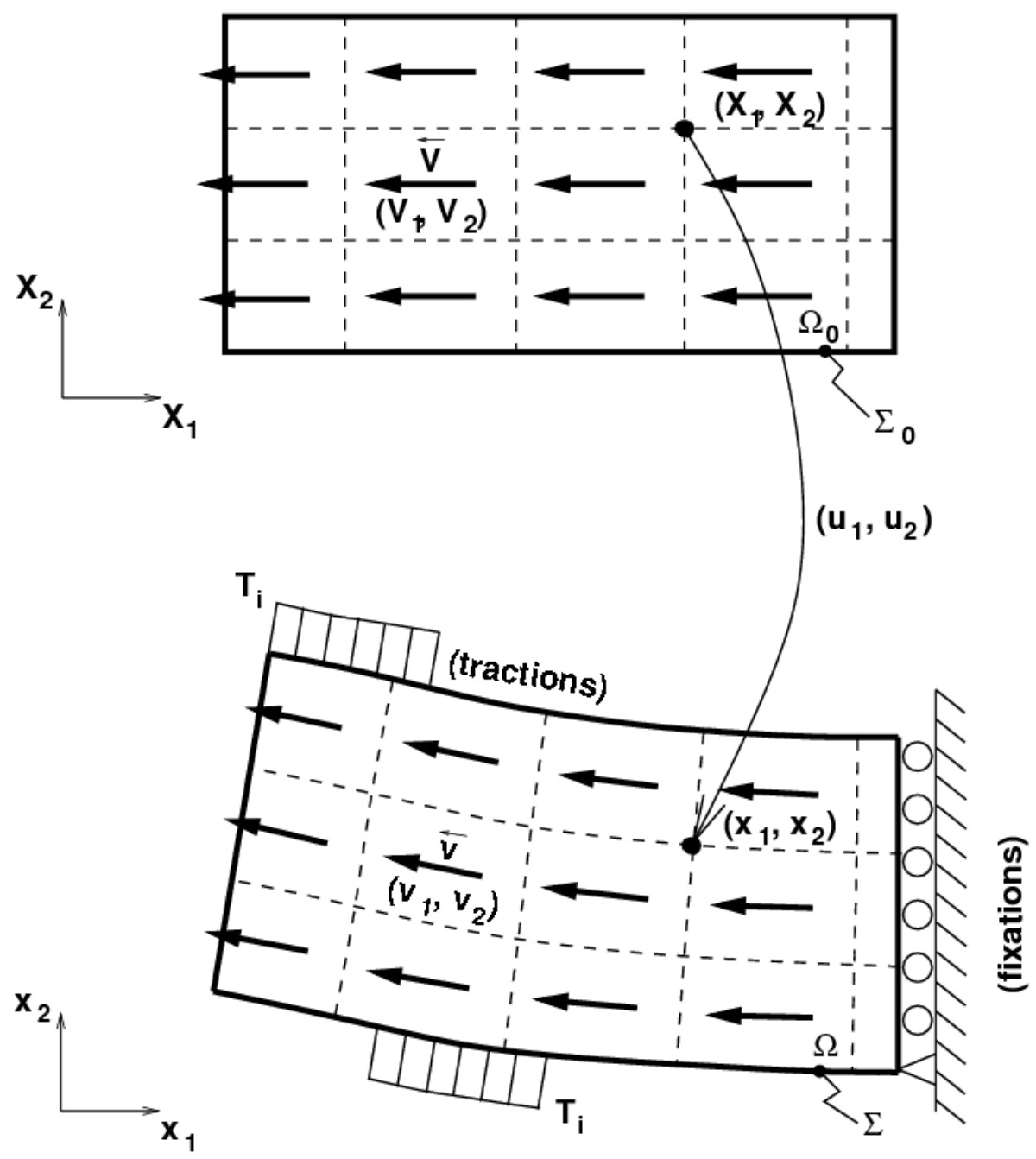

Figure 8. The definition of the (a) undeformed, and (b) deformed configurations and associated quantities involved in the steady-state formulation 

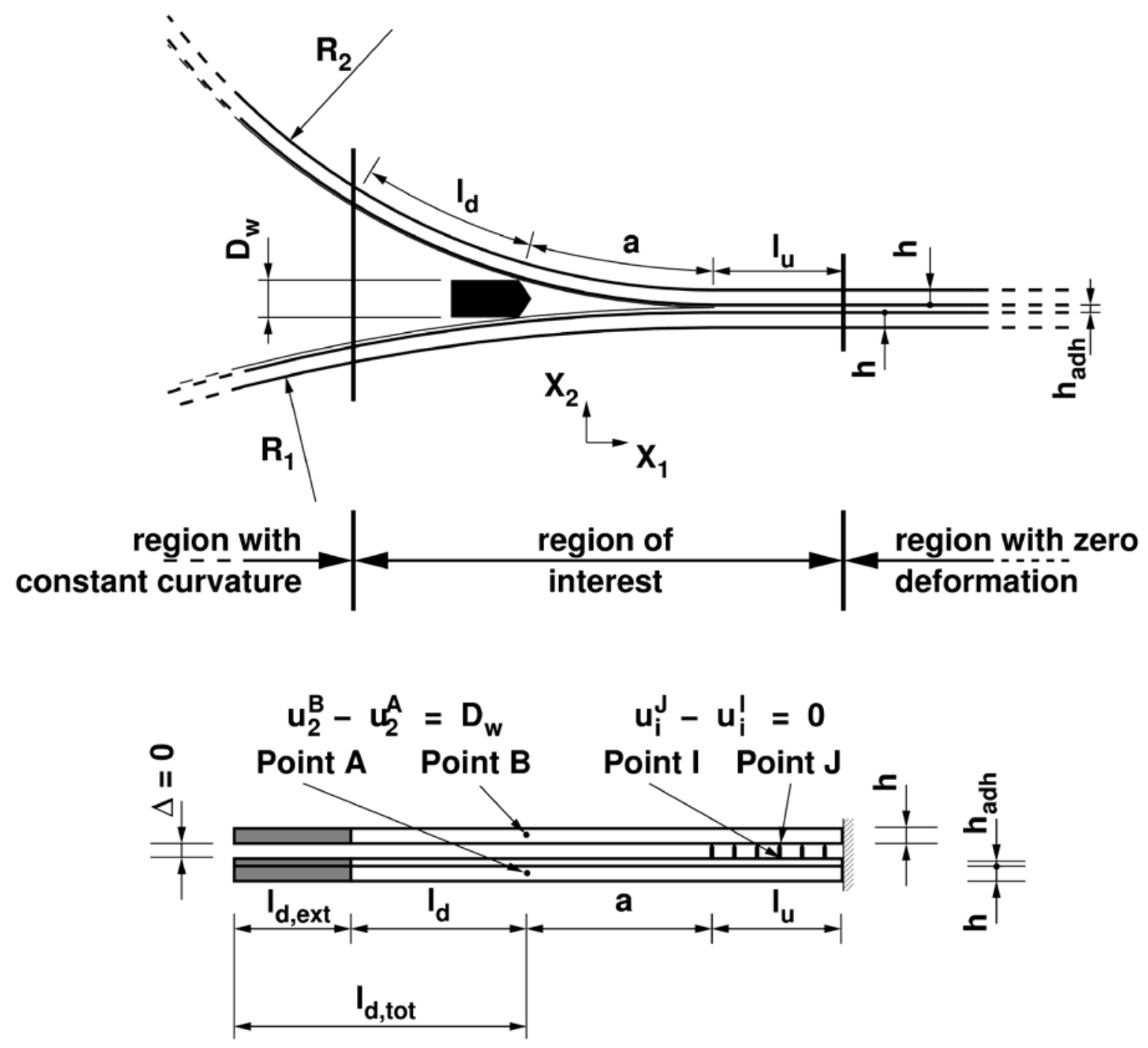

Figure 9. A schematic representation of the wedge-peel test specimen and corresponding boundary conditions used in the numerical-modelling studies 


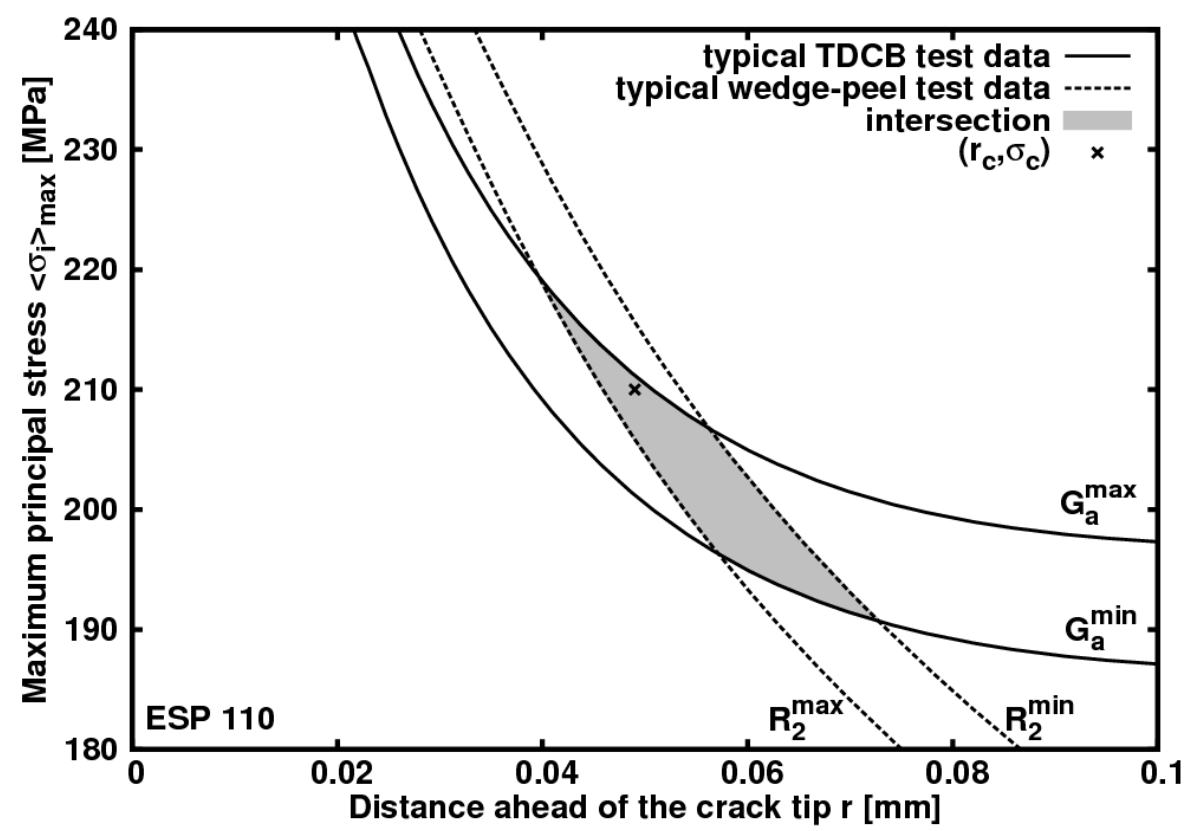

Figure 10. A schematic representation, for the particular case of the adhesive 'ESP 110', of the procedure that is followed to identify the material parameters $\left(r_{c}, \sigma_{c}\right)$ entering the failure criterion 


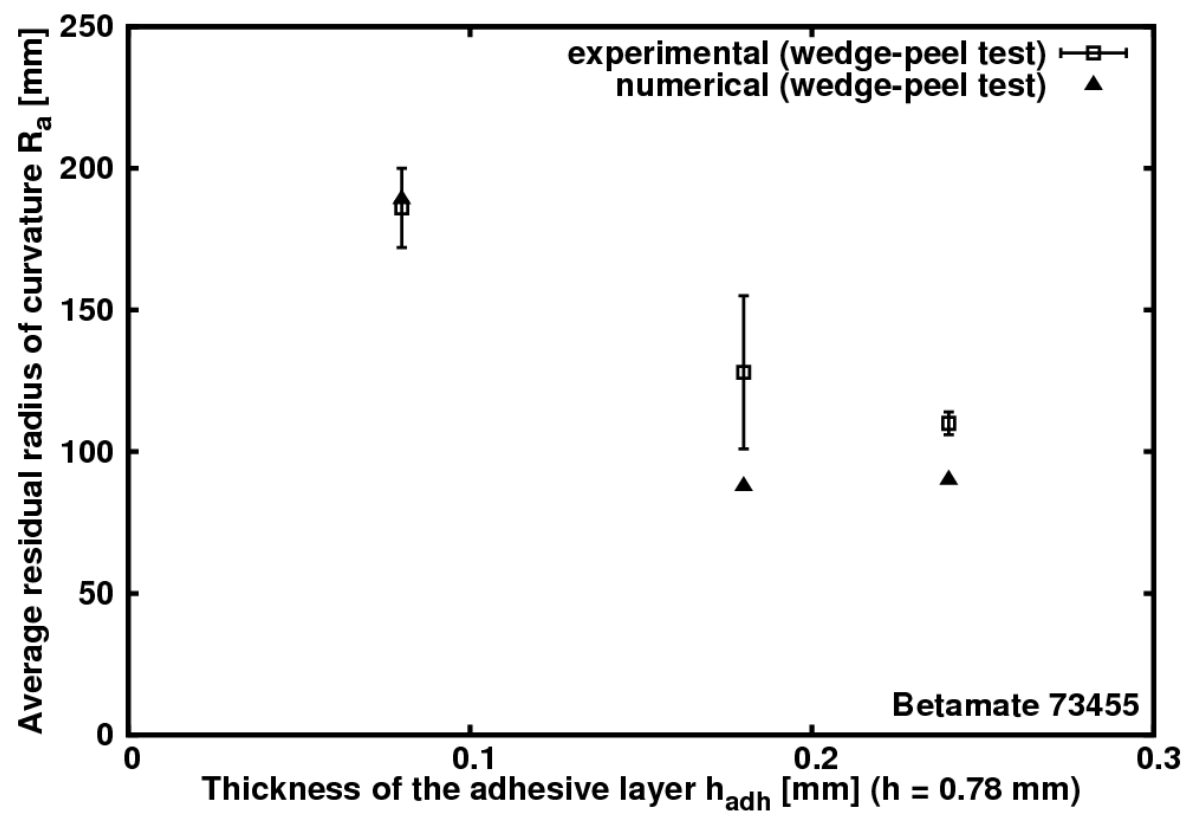

(a)

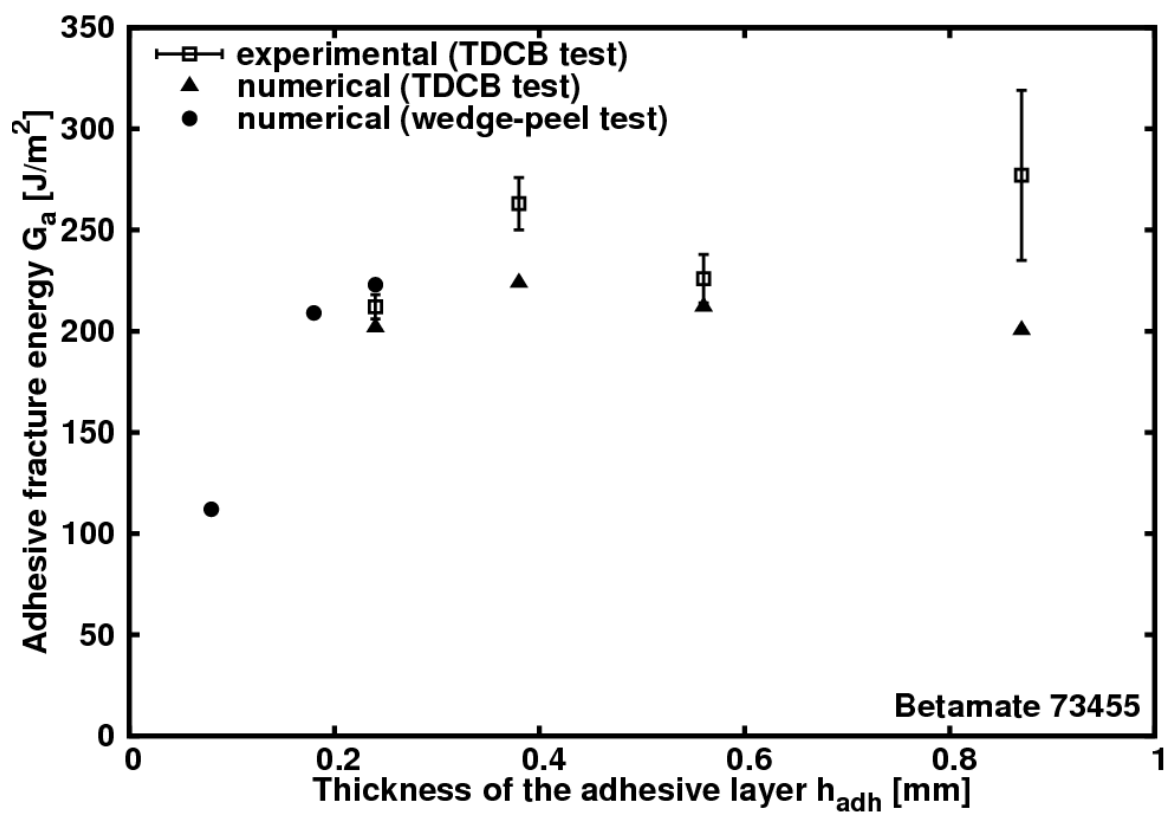

(b)

Figure 11. Comparison between the experimental data and the numerical predictions for the adhesive 'Betamate 73455' obtained (a) in the wedge-peel test, and, (b) in the TDCB test 


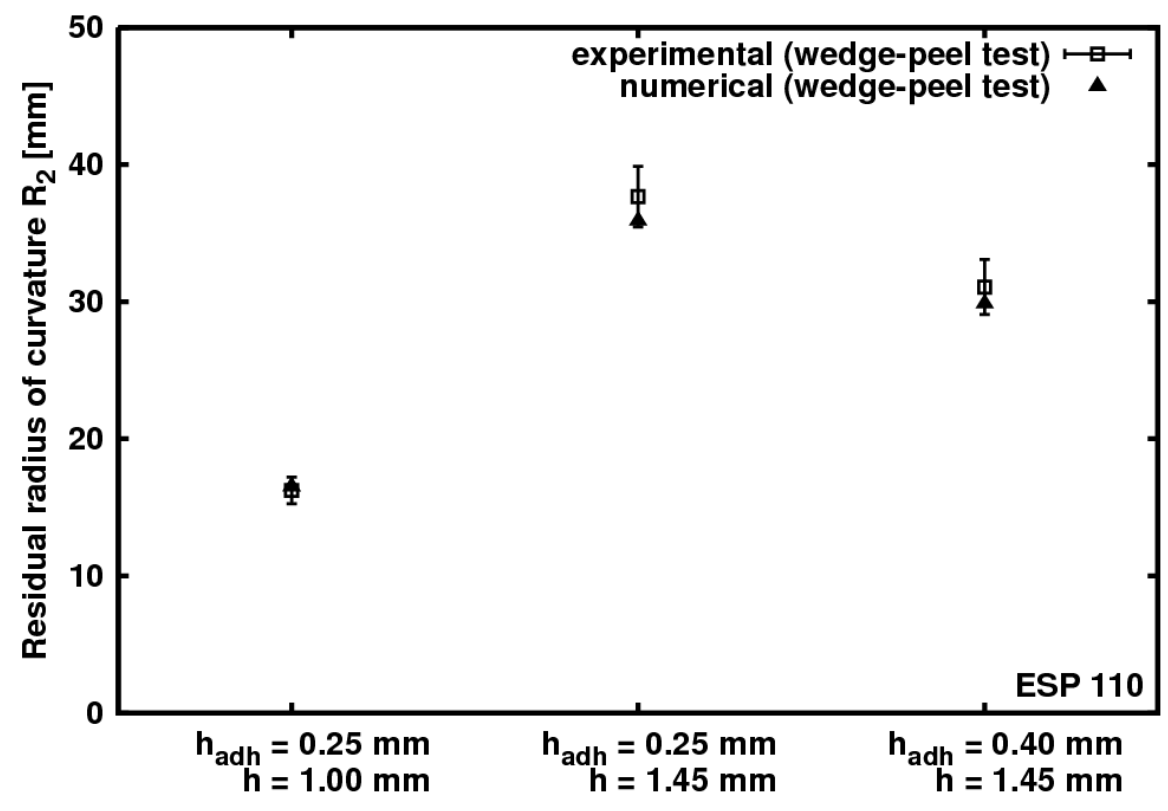

(a)

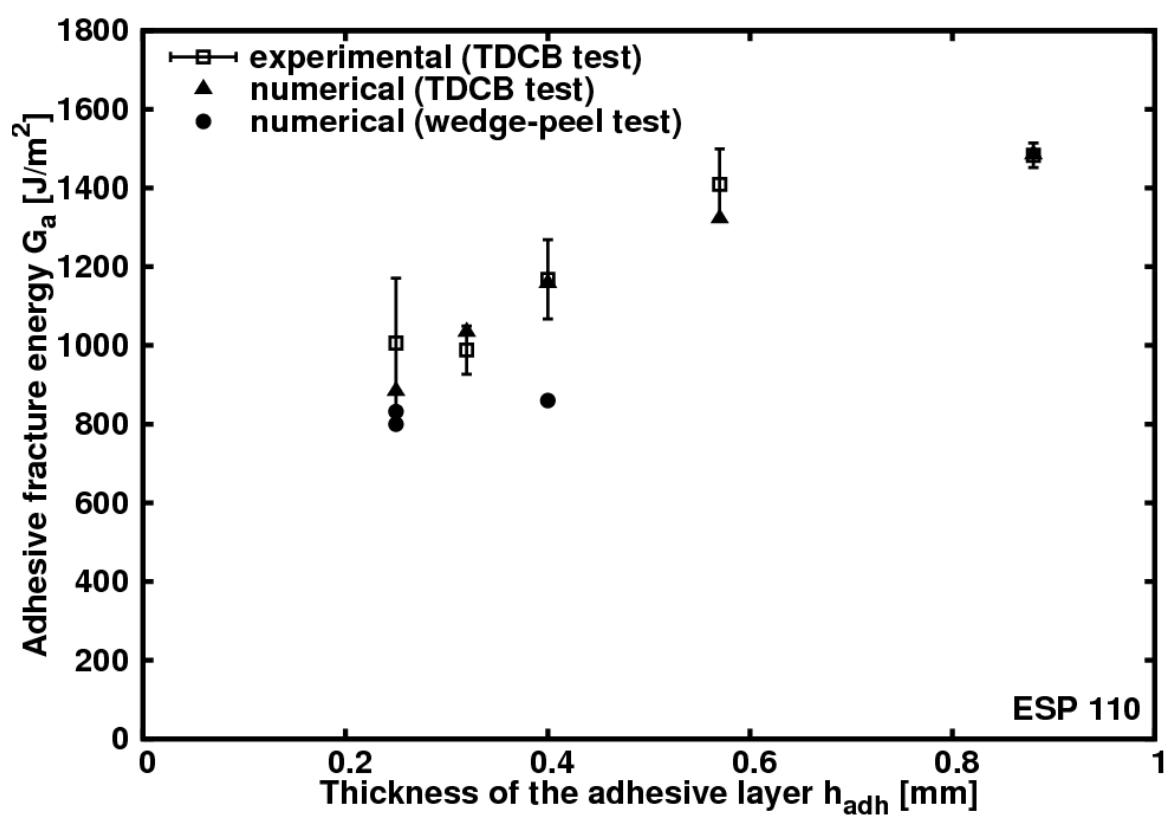

(b)

Figure 12. Comparison between the experimental data and the numerical predictions for the adhesive 'ESP 110' obtained (a) in the wedge-peel test, and, (b) in the TDCB test 


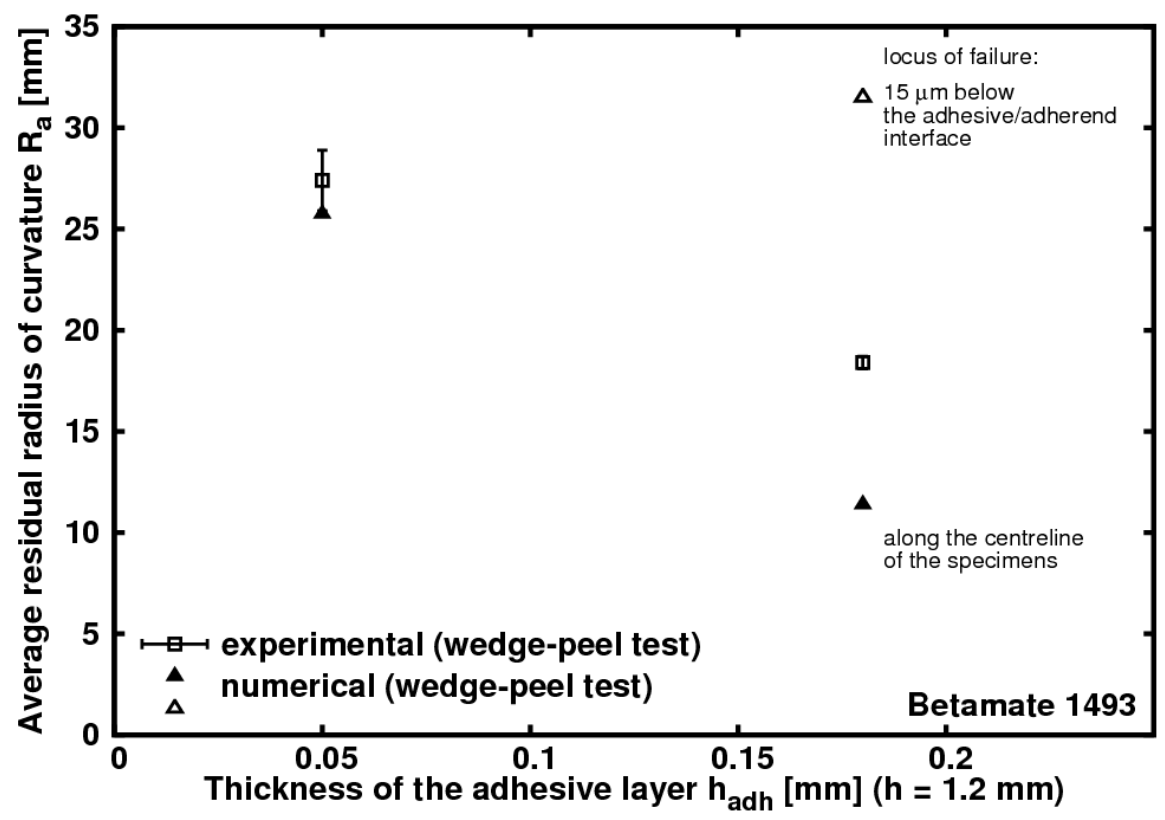

(a)

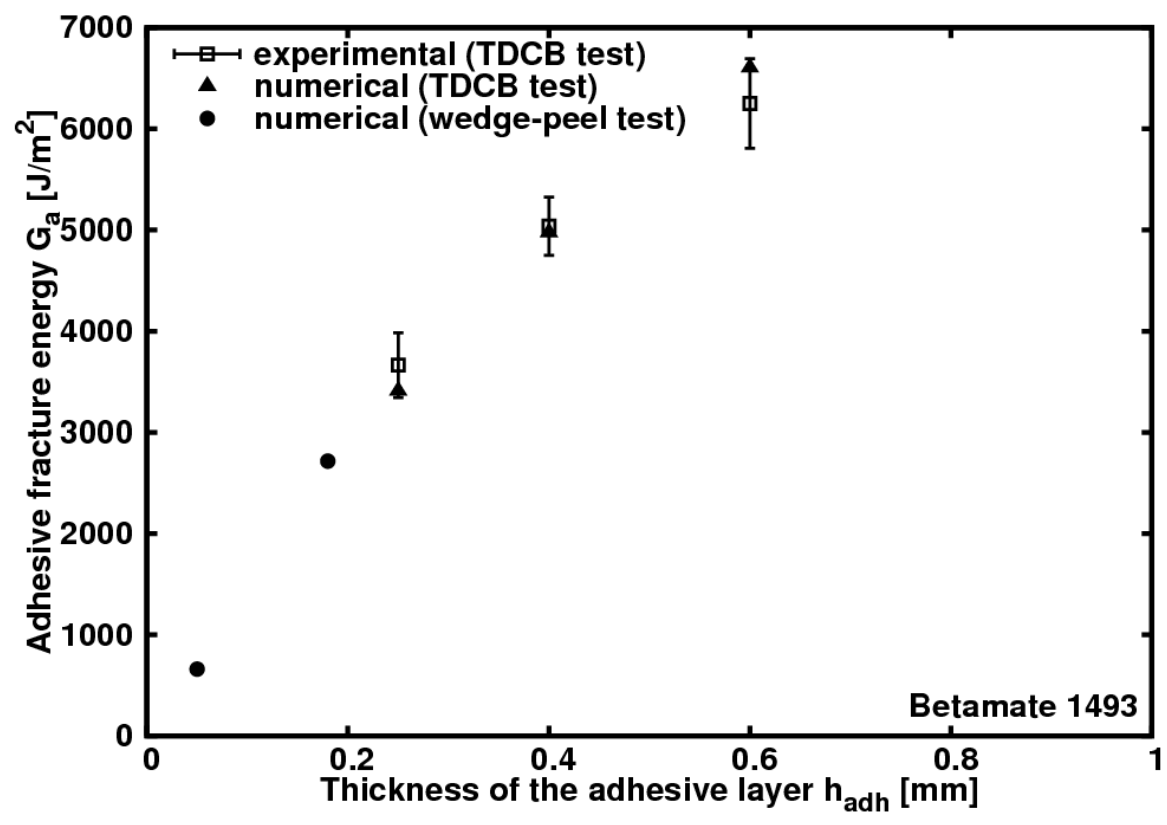

(b)

Figure 13. Comparison between the experimental data and the numerical predictions for the adhesive 'Betamate 1493' obtained (a) in the wedge-peel test, and, (b) in the TDCB test 


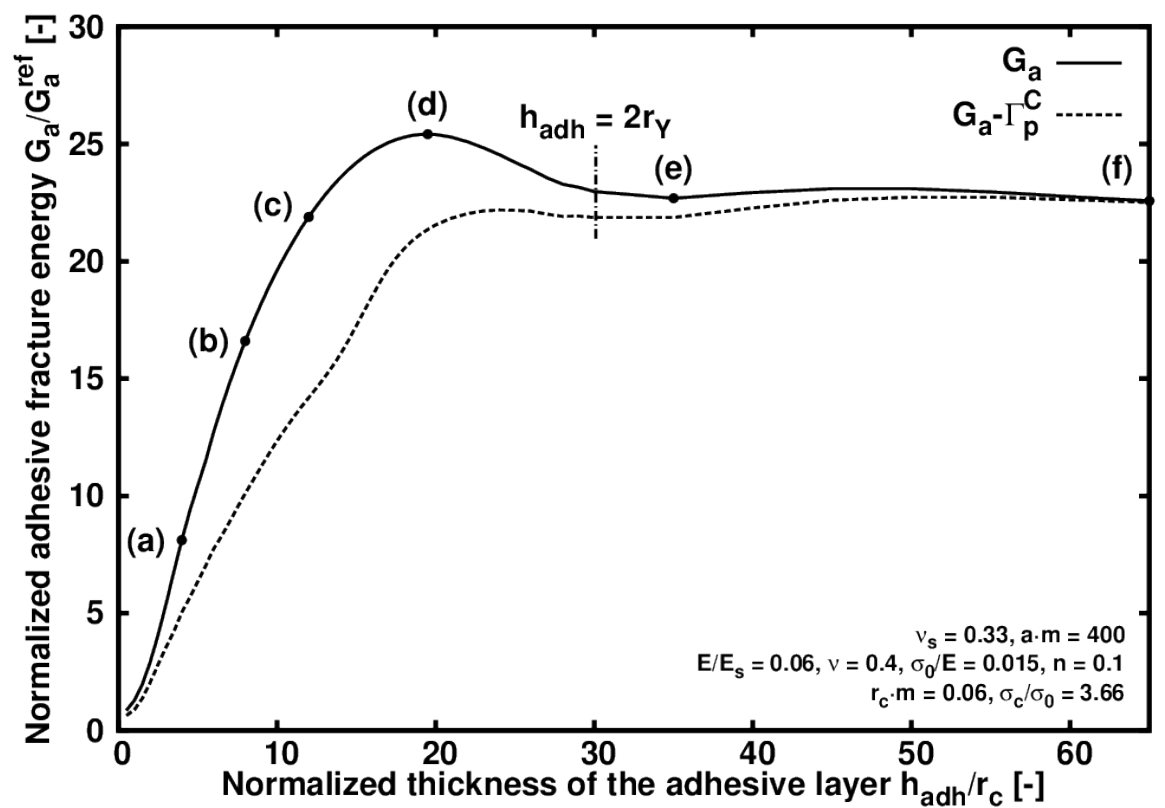

Figure 14. The predicted normalized adhesive fracture energy as a function of the normalized thickness of the adhesive layer for a typical case 


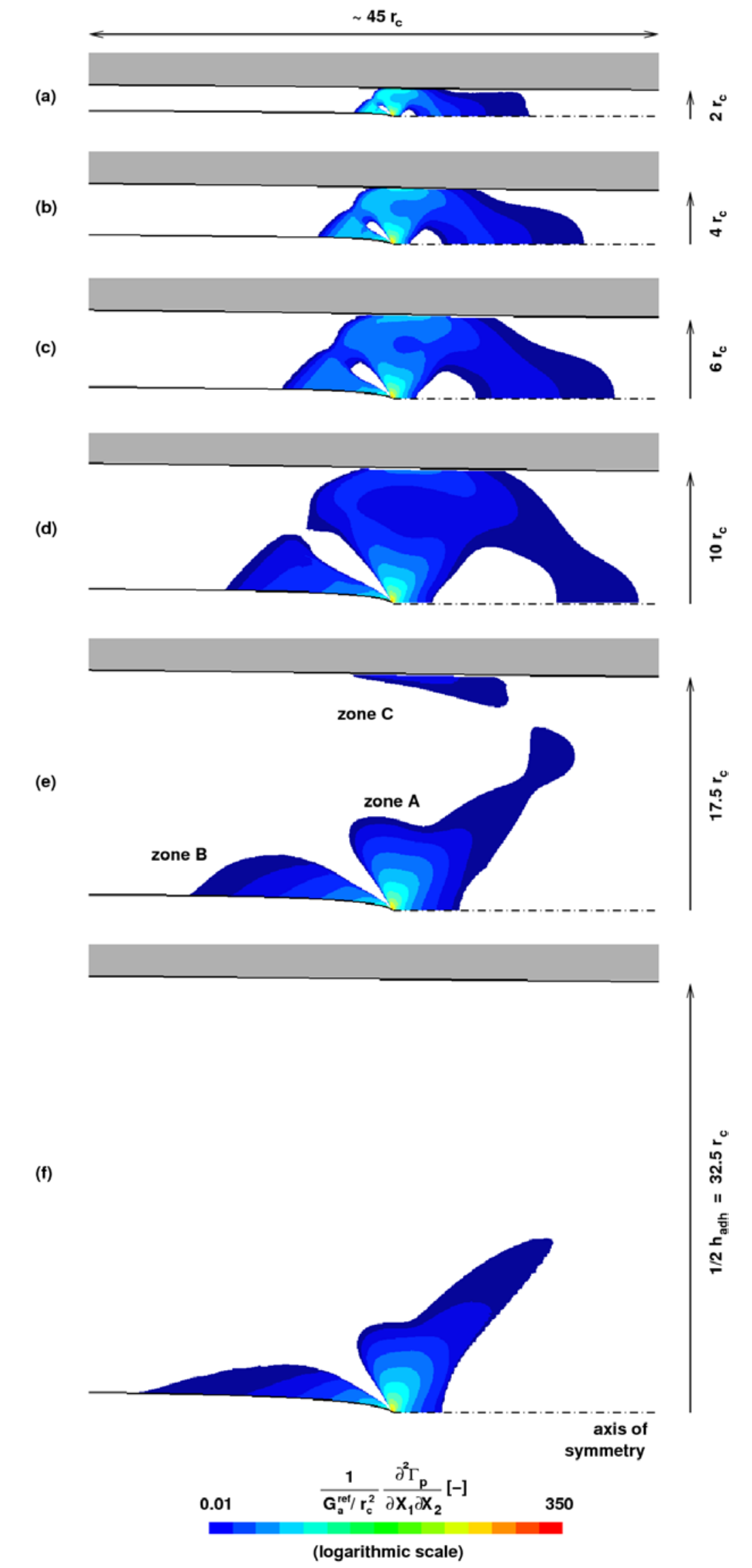

Figure 15. The predicted spatial distribution of the normalized plastic-energy dissipation for different adhesive layer thicknesses 


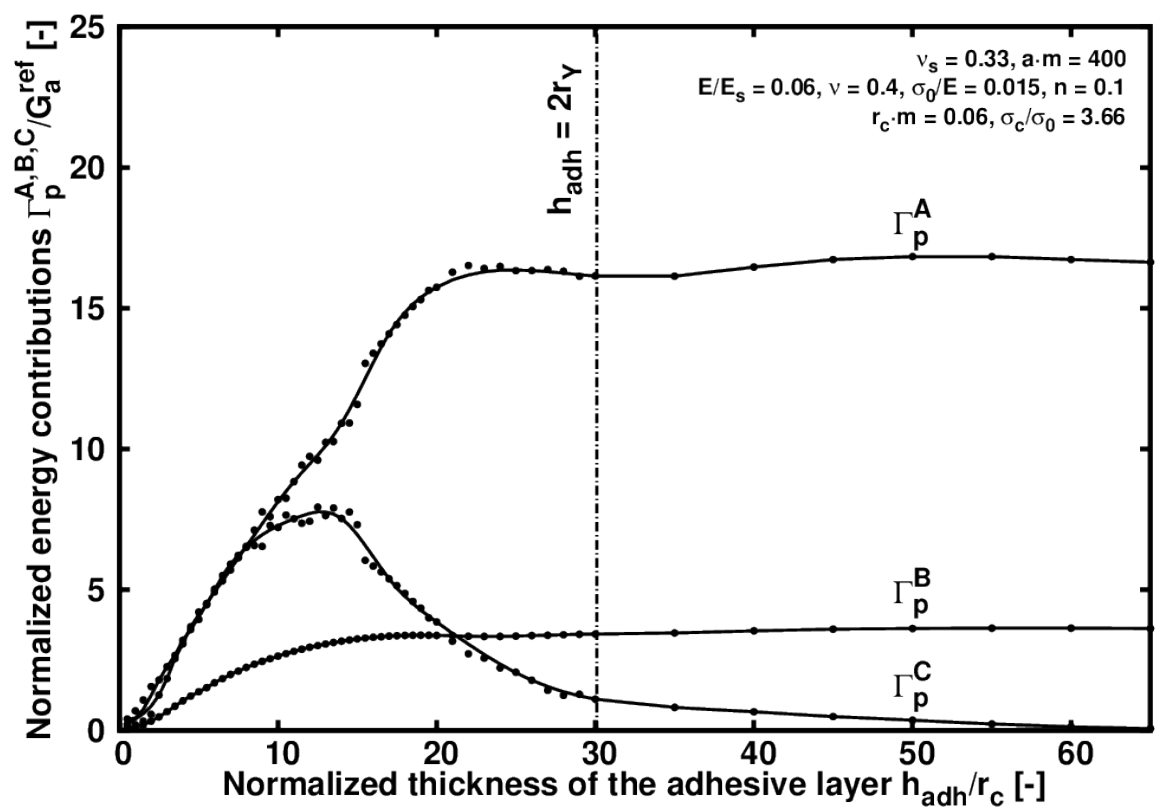

Figure 16. The predicted normalized plastic dissipation contributions to the adhesive fracture energy as a function of the normalized thickness of the adhesive layer for a typical case 


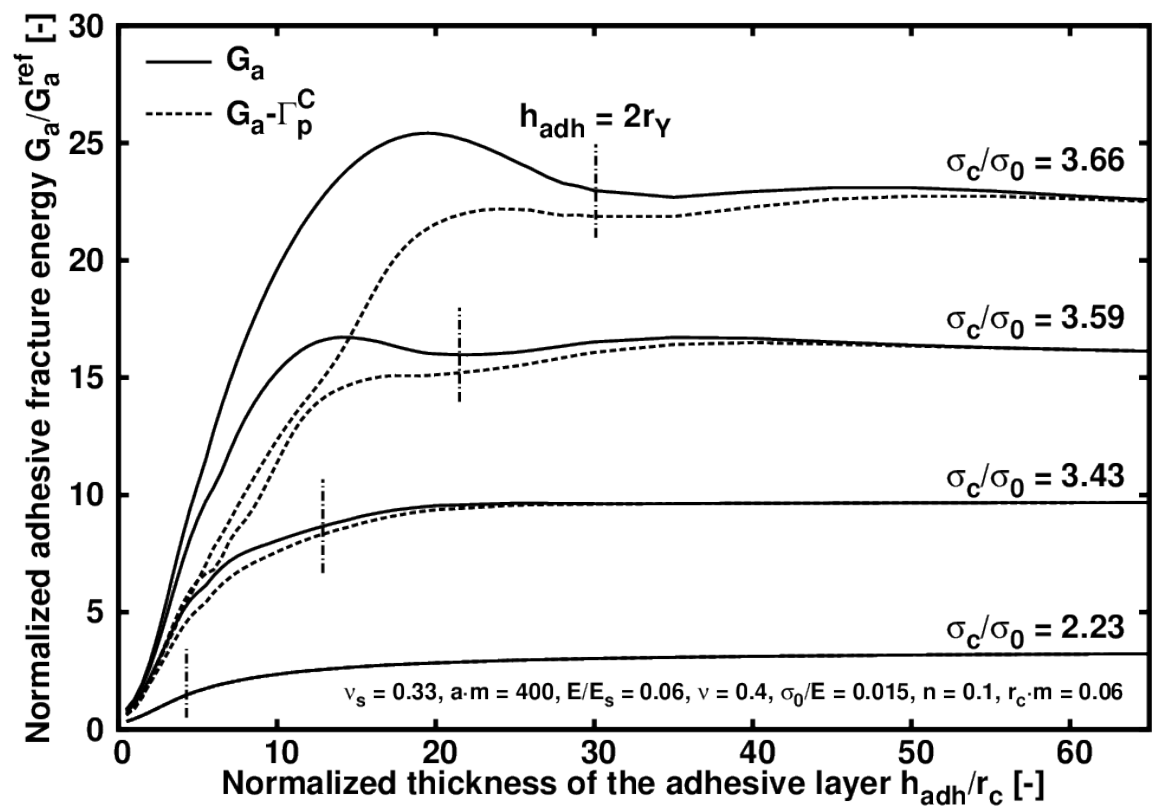

Figure 17. The predicted normalized adhesive fracture energy as a function of the normalized thickness of the adhesive layer for different values of the critical stress 


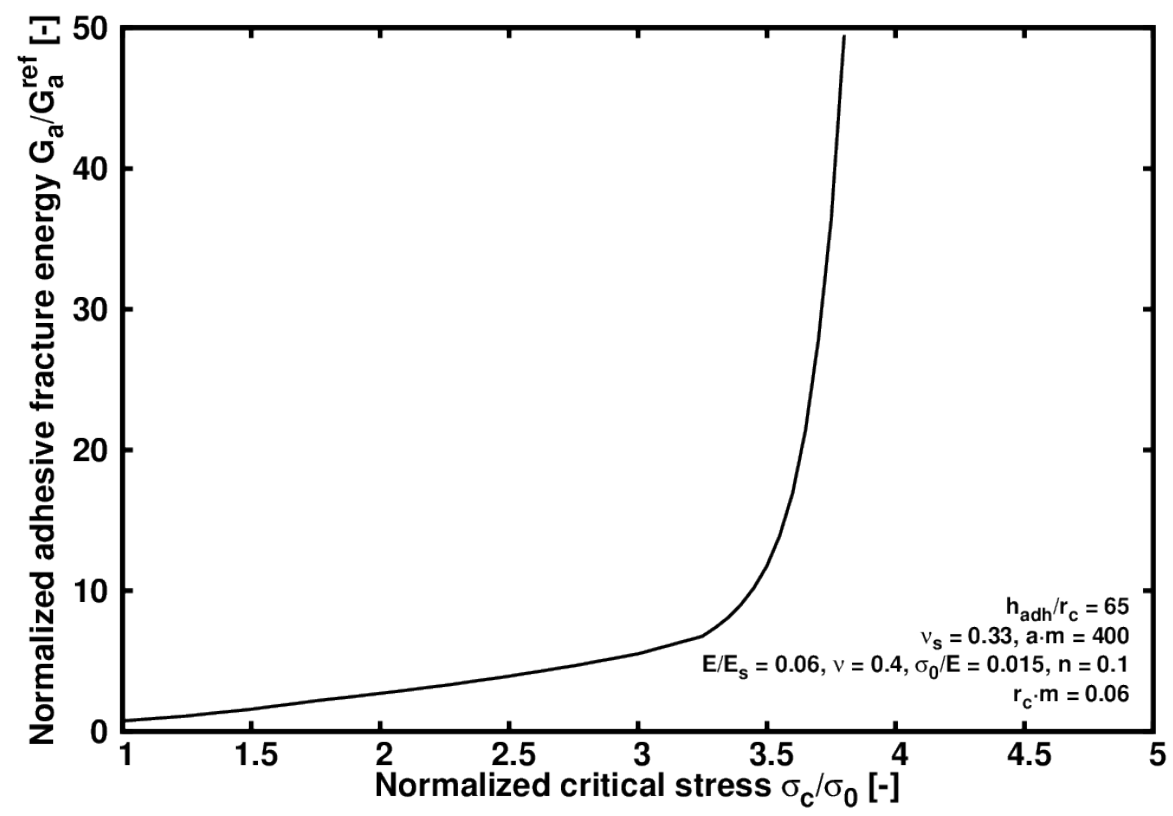

Figure 18. The predicted normalized adhesive fracture energy as a function of the critical stress for large adhesive layer thicknesses 


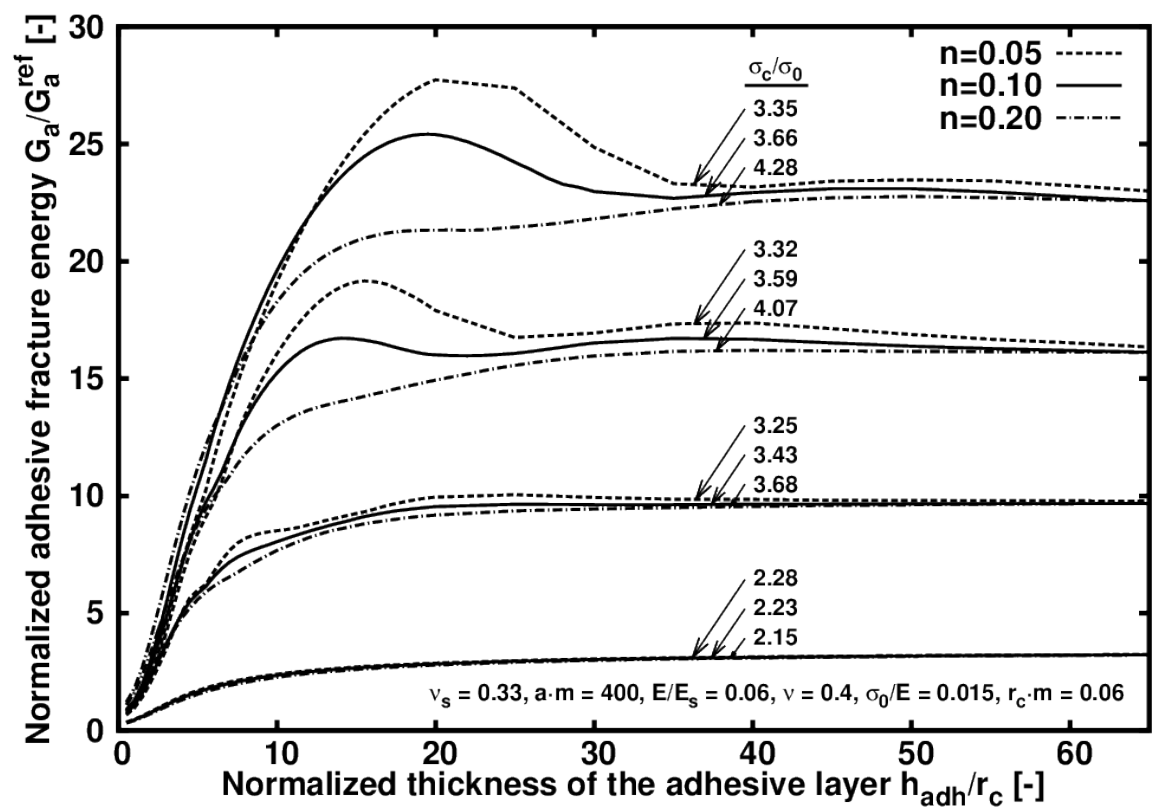

Figure 19. The predicted normalized adhesive fracture energy as a function of the normalized thickness of the adhesive layer for different values of the critical stress and of the hardening exponent 


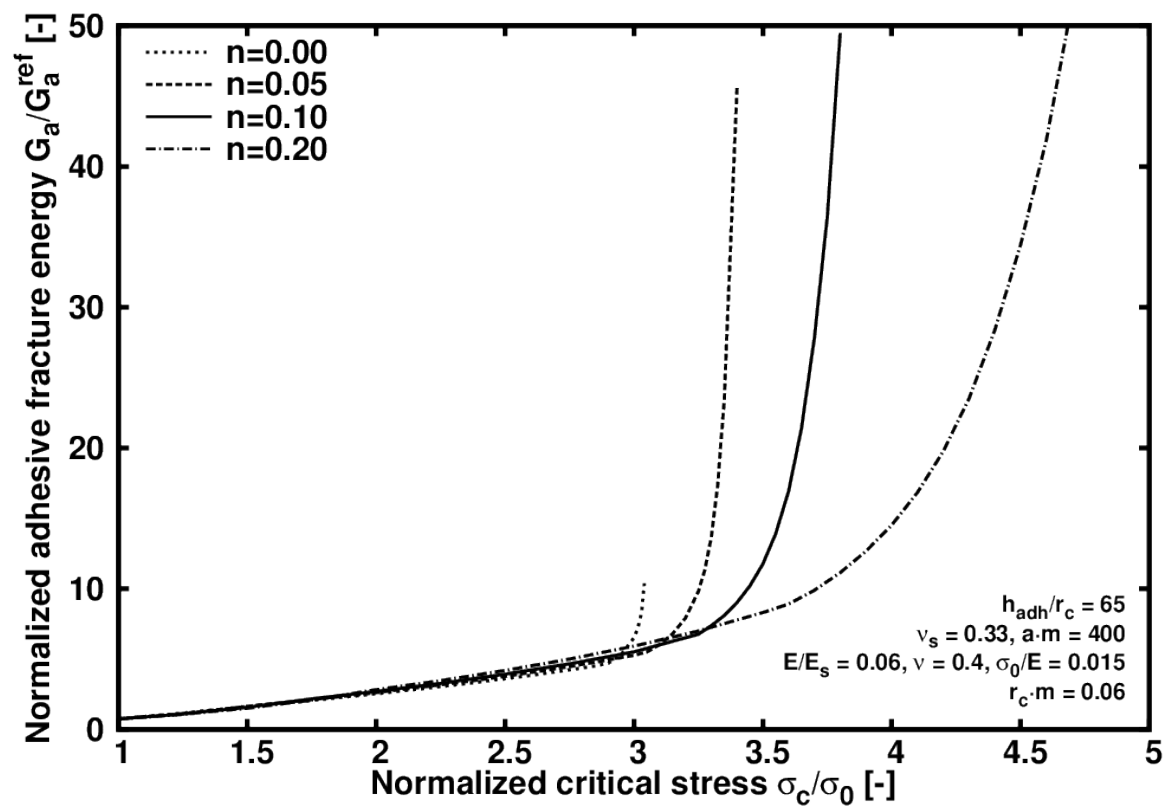

Figure 20. The predicted normalized adhesive fracture energy as a function of the critical stress for large adhesive layer thicknesses and for different values of the hardening exponent 


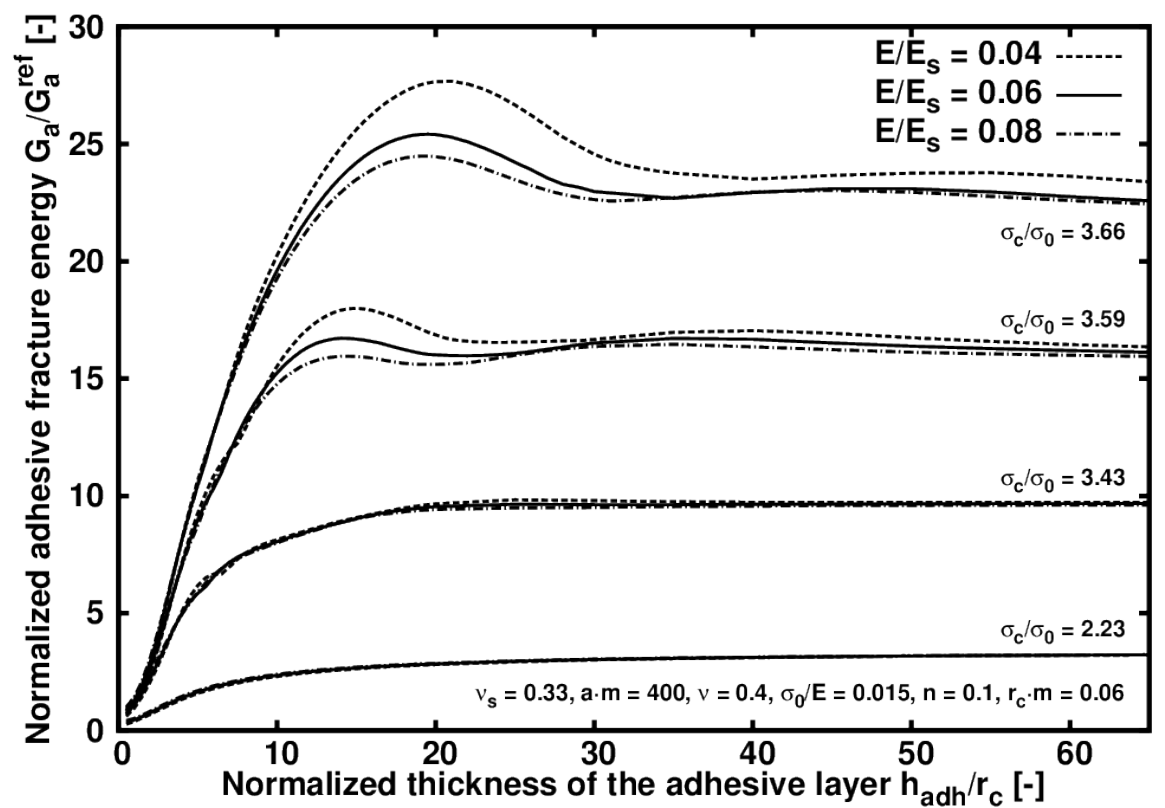

Figure 21. The predicted normalized adhesive fracture energy as a function of the normalized thickness of the adhesive layer for different values of the critical stress and of the Young modulus of the adhesive 


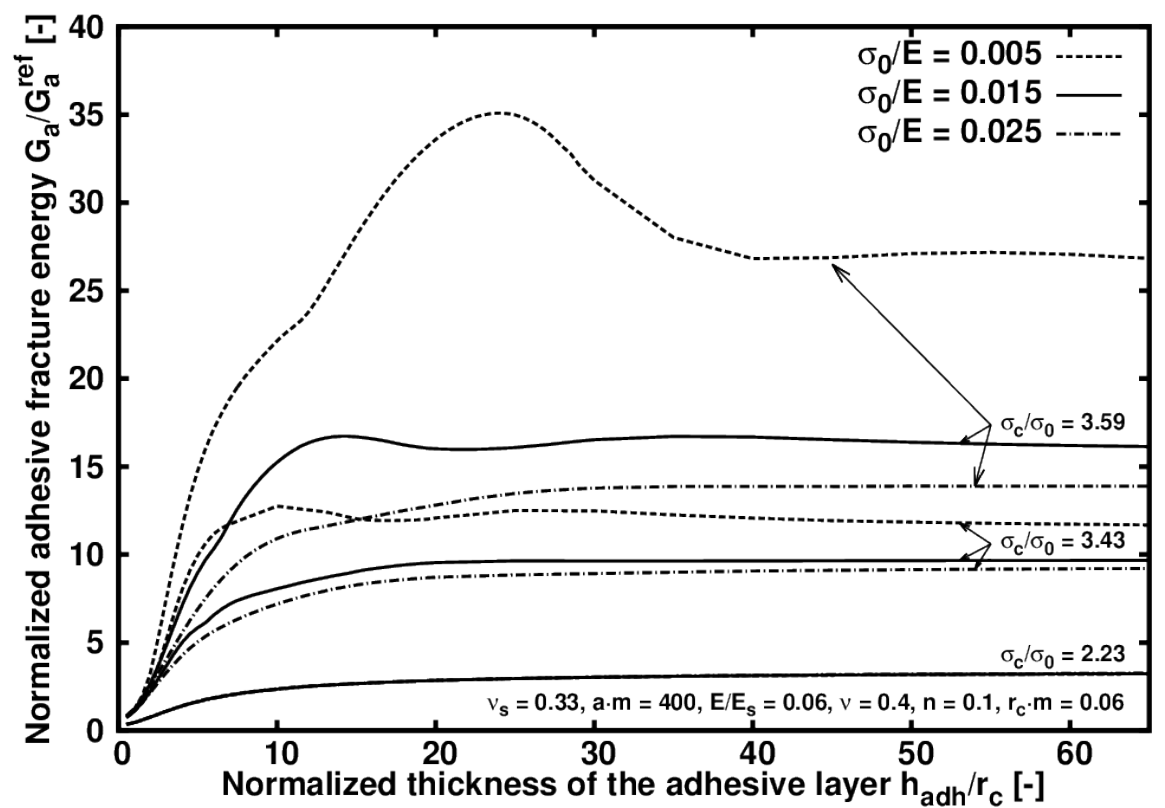

Figure 22. The predicted normalized adhesive fracture energy as a function of the normalized thickness of the adhesive layer for different values of the critical stress and of the initial yield stress of the adhesive 


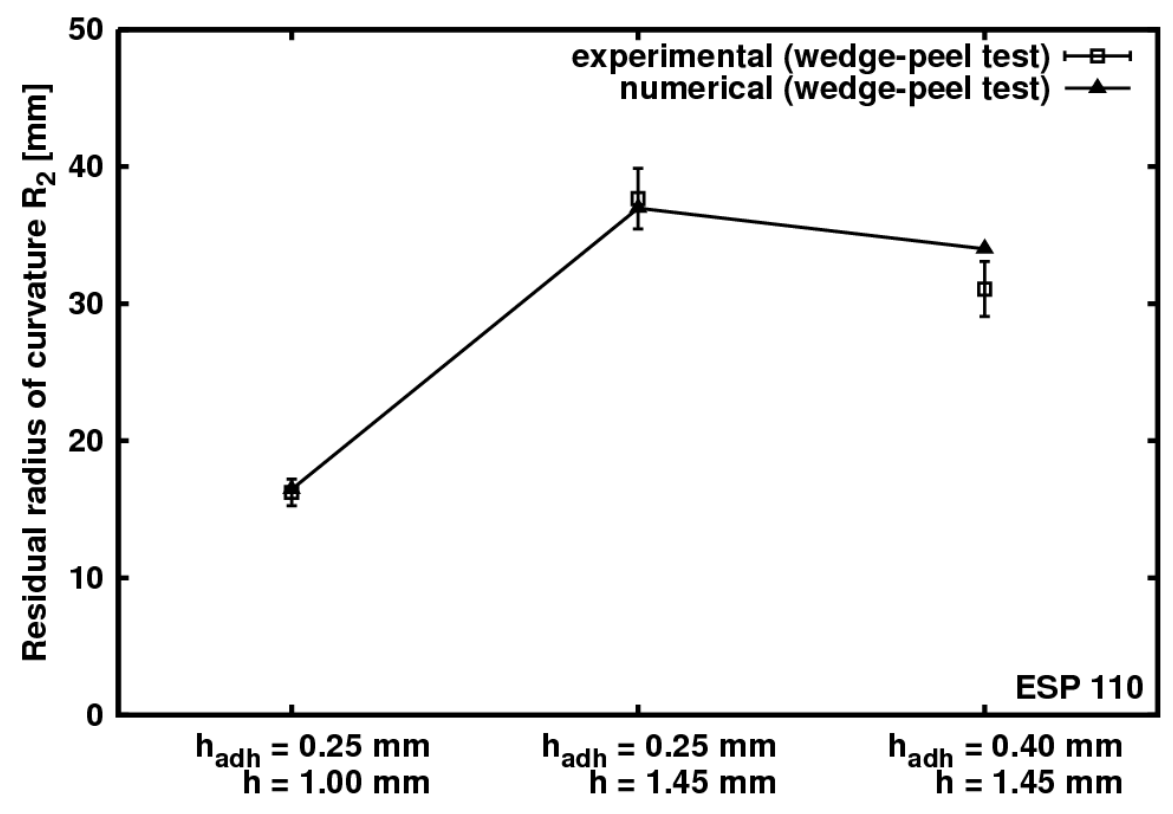

(a)

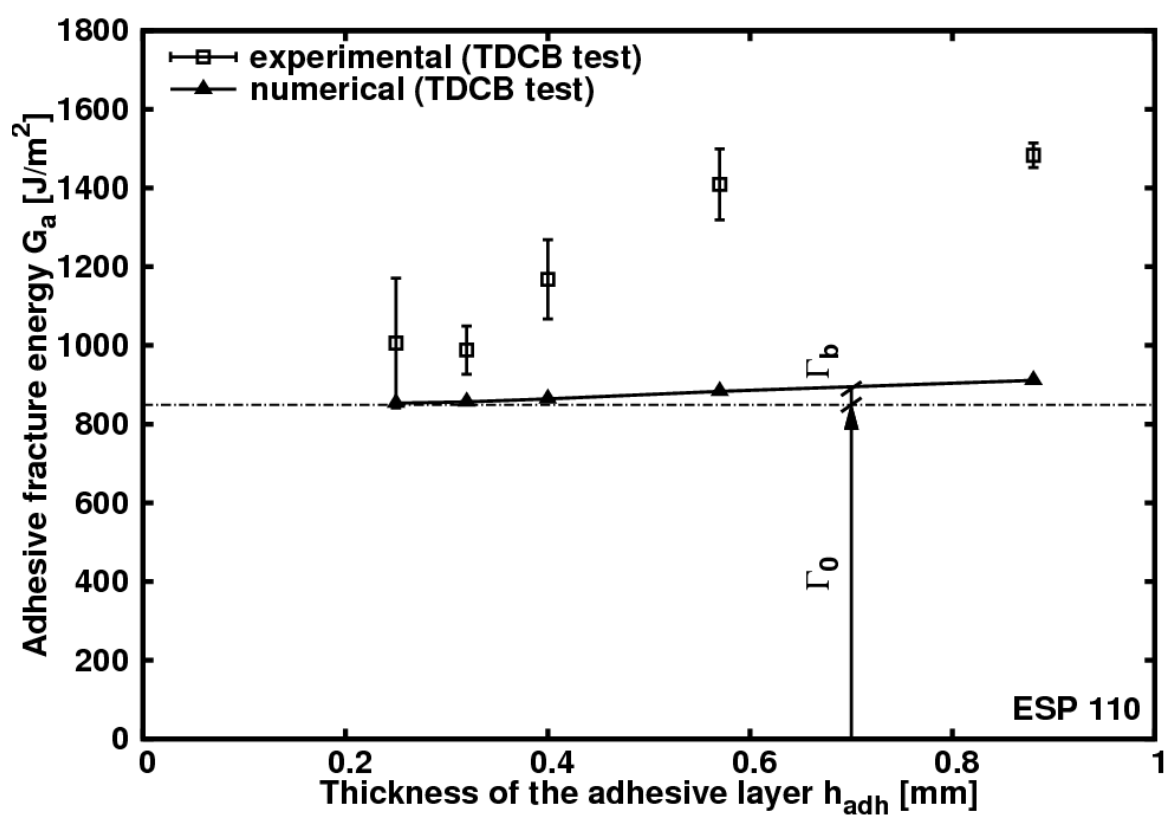

(b)

Figure 23. Comparison between the experimental data and the numerical predictions for the adhesive 'ESP 110' obtained with the CZM and the material parameters [2], (a) in the wedge-peel test, and, (b) in the TDCB test 


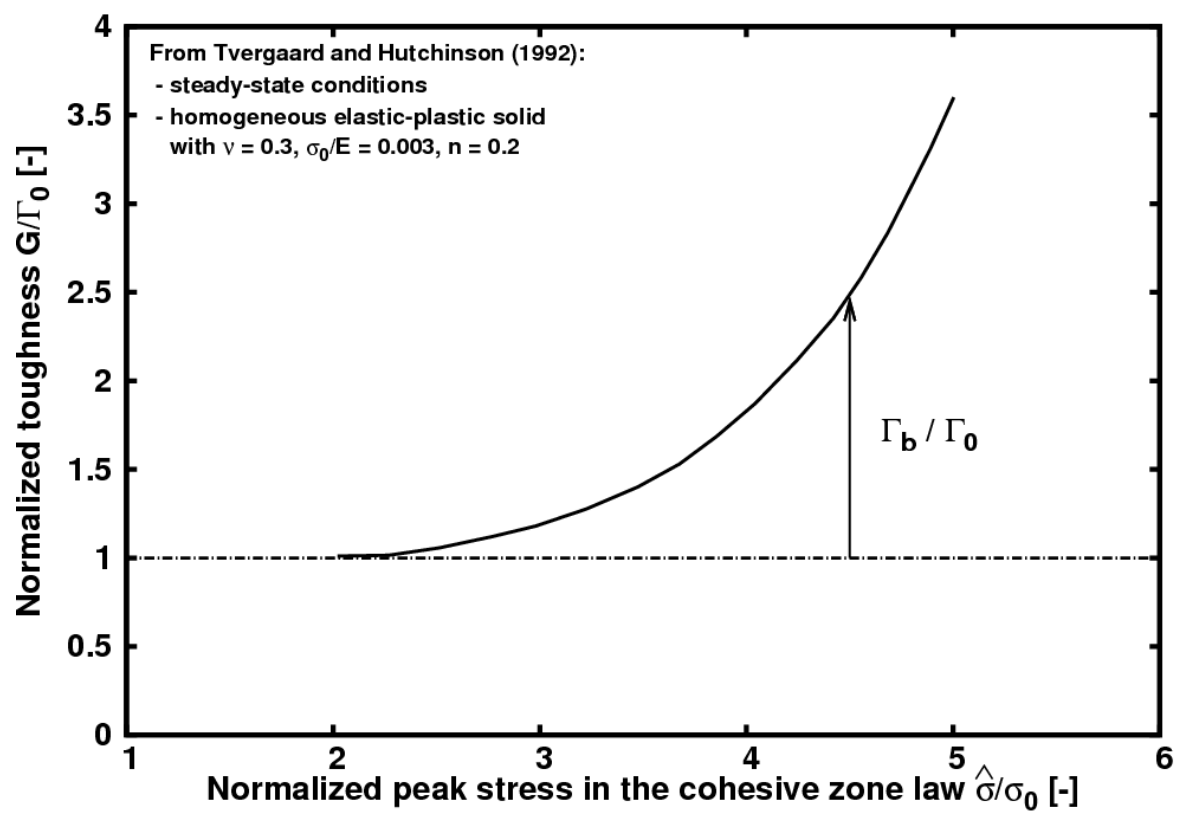

Figure 24. The normalized steady-state toughness of an homogeneous elastic-plastic solid predicted by Tvergaard and Hutchinson [37], as a function of the peak stress in the cohesive zone law, with a model similar to the one that was used by Martiny et al. [2] 


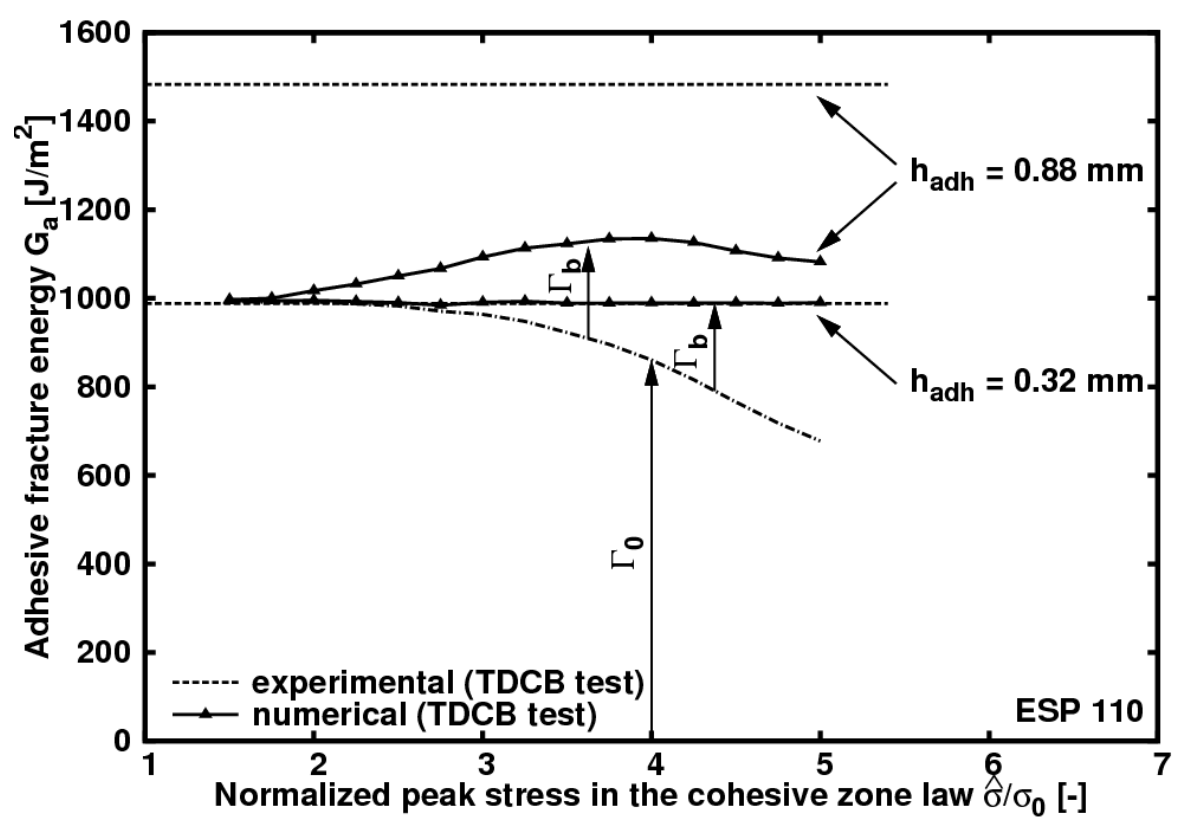

Figure 25. The values of the adhesive fracture energy predicted at an adhesive layer thickness equal to $h_{\text {adh }}=0.88 \mathrm{~mm}$ by the model of Martiny et al. [2] when letting the peak stress in the cohesive zone vary and modifying the value of $\Gamma_{0}$ to reproduce numerically the value of the adhesive fracture energy that was measured experimentally at an adhesive layer thickness of $h_{a d h}=0.32 \mathrm{~mm}$ 
Table 1. Experimental LEFM TDCB test results for the adhesive 'Betamate 73455' (adherend: aluminium alloy; locus of failure: close to the centreline of the specimens)

\begin{tabular}{ccc}
\hline$h_{a d h}[\mathrm{~mm}]$ & \multicolumn{2}{c}{$G_{a}\left[\mathrm{~J} / \mathrm{m}^{2}\right]$} \\
\hline 0.24 & $212 \pm 4$ \\
0.38 & $263 \pm 13$ \\
0.56 & $226 \pm 12$ \\
0.87 & $277 \pm 42$ \\
\hline
\end{tabular}


Table 2. Experimental EPFM wedge-peel test results for the adhesive 'Betamate 73455' (mild-steel adherend thickness, $h=0.78 \mathrm{~mm}$;

locus of failure: close to the centreline of the specimens)

\begin{tabular}{cccc}
\hline$h_{a d h}[\mathrm{~mm}]$ & \multicolumn{3}{c}{$R_{a}[\mathrm{~mm}]$} \\
\hline 0.08 & $186 \quad \pm \quad 14$ \\
0.18 & $128 \quad \pm \quad 27$ \\
0.24 & $110 \pm 4$ & 4 \\
\hline
\end{tabular}


Table 3. Experimental LEFM TDCB test results for the adhesive 'ESP 110' (adherend: aluminium alloy; locus of failure: close to the centreline of the specimens)

\begin{tabular}{crcc}
\hline$h_{\text {adh }}[\mathrm{mm}]$ & \multicolumn{3}{c}{$G_{a}\left[\mathrm{~J} / \mathrm{m}^{2}\right]$} \\
\hline 0.25 & 1006 & \pm & 165 \\
0.32 & 988 & \pm & 61 \\
0.40 & 1168 & \pm & 101 \\
0.57 & 1409 & \pm & 90 \\
0.88 & 1483 & \pm & 31 \\
\hline
\end{tabular}


Table 4. Experimental EPFM wedge-peel test results for the adhesive 'ESP 110' (adherend: aluminium alloy; locus of failure: close to one of the adhesive/adherend interfaces)

\begin{tabular}{ccc}
\hline$h_{a d h}[\mathrm{~mm}]$ & $h[\mathrm{~mm}]$ & $R_{2}[\mathrm{~mm}]$ \\
\hline 0.25 & 1.00 & $186 \pm 14$ \\
0.25 & 1.45 & $128 \pm 27$ \\
0.40 & 1.45 & $110 \pm 4$ \\
\hline
\end{tabular}


Table 5. Experimental LEFM TDCB test results for the adhesive 'Betamate 1493' (adherend: aluminium alloy; locus of failure: close to the centreline of the specimens)

\begin{tabular}{llll}
\hline $\begin{array}{c}h_{\text {adh }} \\
{[\mathrm{mm}]}\end{array}$ & & $G_{a}\left[\mathrm{~J} / \mathrm{m}^{2}\right]$ \\
\hline 0.25 & 3665 & \pm & 321 \\
0.40 & 5037 & \pm & 287 \\
0.60 & 6250 & \pm & 441 \\
\hline
\end{tabular}


Table 6. Experimental EPFM wedge-peel test results for the adhesive 'Betamate 1493' (mild-steel adherend thickness, $h=1.2 \mathrm{~mm}$; locus of failure: close to the centreline of the specimens)

\begin{tabular}{cccc}
\hline$h_{\text {adh }}[\mathrm{mm}]$ & \multicolumn{3}{c}{$R_{a}[\mathrm{~mm}]$} \\
\hline 0.05 & $27.4 \quad \pm$ & 1.5 \\
0.18 & 18.4 & \pm & 0.3 \\
\hline
\end{tabular}


Table 7. Elastic-plastic properties used in the model

\begin{tabular}{|c|c|c|c|c|c|}
\hline \multicolumn{6}{|c|}{ Elastic behaviour } \\
\hline Material & \multirow{2}{*}{$\frac{E[\mathrm{GPa}]}{72.4}$} & \multicolumn{4}{|l|}{$v[-]$} \\
\hline AA 2014A & & \multicolumn{4}{|l|}{0.33} \\
\hline \multicolumn{6}{|c|}{ Elastic-plastic behaviour fitted using Equation (14) } \\
\hline Material & $E[\mathrm{GPa}]$ & $v[-]$ & $\sigma_{0}[\mathrm{MPa}]$ & $n[-]$ & \\
\hline Mild steel & 210 & 0.30 & 124 & 0.14 & \\
\hline \multicolumn{6}{|c|}{ Elastic-plastic behaviour fitted using Equation (15) } \\
\hline Material & $E[\mathrm{GPa}]$ & $v[-]$ & $\kappa[\mathrm{MPa}]$ & $\eta[-]$ & $q[-]$ \\
\hline AA 5754-O (1 mm) & 74.7 & 0.33 & 114 & 87.5 & 0.29 \\
\hline AA $5754-0(1.45 \mathrm{~mm})$ & 79.5 & 0.33 & 112 & 107 & 0.257 \\
\hline 'Betamate 73455’ & 6 & 0.45 & 13 & 87000 & 0.13 \\
\hline 'ESP 110’ & 5.72 & 0.40 & 5.34 & 210000 & 0.32 \\
\hline 'Betamate 1493' & 1.8 & 0.45 & 20.4 & 19200 & 0.092 \\
\hline
\end{tabular}


Table 8. Failure properties used in the model

\begin{tabular}{lcccc}
\hline Adhesive & $\begin{array}{c}r_{c} \\
{[\mu \mathrm{m}]}\end{array}$ & $\begin{array}{c}\sigma_{c} \\
{[\mathrm{MPa}]}\end{array}$ & $\begin{array}{c}\text { Particle } \\
\text { size }[\mu \mathrm{m}]\end{array}$ & $\begin{array}{c}\text { Particle } \\
\text { spacing }[\mu \mathrm{m}]\end{array}$ \\
\hline 'Betamate 73455' & $18 \pm 3$ & $98 \pm 0.4$ & $10-200$ & $20-200$ \\
'ESP 110' & $49 \pm 3$ & $210 \pm 3.3$ & $30-100$ & $50-100$ \\
'Betamate 1493' & $6.8 \pm 1$ & $141 \pm 1.0$ & $0.1-5$ & $1-10$ \\
\hline
\end{tabular}


Table 9. Mechanical properties of the three adhesives fitted using Equation (14)

\begin{tabular}{lcccccc} 
Adhesive & $E[\mathrm{GPa}]$ & $v[-]$ & $\sigma_{0}[\mathrm{MPa}]$ & $n[-]$ & $\sigma_{c} / \sigma_{0}[-]$ & $G_{a}^{r e f}\left[\mathrm{~J} / \mathrm{m}^{2}\right]$ \\
\hline 'Betamate 73455' & 6 & 0.45 & 19.6 & 0.41 & 5.0 & 5.8 \\
'ESP 110' & 5.72 & 0.40 & 28.0 & 0.54 & 7.5 & 35.4 \\
'Betamate 1493' & 1.8 & 0.45 & 32.1 & 0.12 & 4.4 & 19.5 \\
\hline
\end{tabular}

